\title{
Precise radial velocities of giant stars
}

\section{A possible 2:1 resonant planet pair around the $\mathrm{K}$ giant star $\eta$ Ceti $^{\star, \star \star}$}

\author{
Trifon Trifonov ${ }^{1}$, Sabine Reffert ${ }^{1}$, Xianyu Tan ${ }^{2,3}$, Man Hoi Lee ${ }^{2,4}$, and Andreas Quirrenbach ${ }^{1}$ \\ 1 ZAH-Landessternwarte, Königstuhl 12, 69117 Heidelberg, Germany \\ e-mail: t.trifonov@lsw . uni-heidelberg.de \\ 2 Department of Earth Sciences, The University of Hong Kong, Pokfulam Road, Hong Kong, PR China \\ 3 Department of Planetary Sciences and Lunar and Planetary Laboratory, The University of Arizona, 1629 University Blvd., Tucson \\ AZ 85721, USA \\ 4 Department of Physics, The University of Hong Kong, Pokfulman Road, Hong Kong, PR China
}

Received 21 October 2013 / Accepted 20 June 2014

\begin{abstract}
We report the discovery of a new planetary system around the $\mathrm{K}$ giant $\eta$ Cet (HIP 5364, HD 6805, HR 334) based on 118 highprecision optical radial velocities taken at Lick Observatory since July 2000. Since October 2011 an additional nine near-infrared Doppler measurements have been taken using the ESO CRIRES spectrograph (VLT, UT1). The visible data set shows two clear periodicities. Although we cannot completely rule out that the shorter period is due to rotational modulation of stellar features, the infrared data show the same variations as in the optical, which strongly supports that the variations are caused by two planets. Assuming the mass of $\eta$ Cet to be $1.7 M_{\odot}$, the best edge-on coplanar dynamical fit to the data is consistent with two massive planets $\left(m_{b} \sin i=2.6 \pm 0.2 M_{\mathrm{Jup}}, m_{c} \sin i=3.3 \pm 0.2 M_{\mathrm{Jup}}\right)$, with periods of $P_{b}=407 \pm 3$ days and $P_{c}=740 \pm 5$ days and eccentricities of $e_{b}=0.12 \pm 0.05$ and $e_{c}=0.08 \pm 0.04$. These mass and period ratios suggest possible strong interactions between the planets, and a dynamical test is mandatory. We tested a wide variety of edge-on coplanar and inclined planetary configurations for stability, which agree with the derived radial velocities. We find that for a coplanar configuration there are several isolated stable solutions and two well defined stability regions. In certain orbital configurations with moderate $e_{b}$ eccentricity, the planets can be effectively trapped in an anti-aligned 2:1 mean motion resonance that stabilizes the system. A much larger non-resonant stable region exists in low-eccentricity parameter space, although it appears to be much farther from the best fit than the $2: 1$ resonant region. In all other cases, the system is categorized as unstable or chaotic. Another conclusion from the coplanar inclined dynamical test is that the planets can be at most a factor of $\sim 1.4$ more massive than their suggested minimum masses. Assuming yet higher inclinations, and thus larger planetary masses, leads to instability in all cases. This stability constraint on the inclination excludes the possibility of two brown dwarfs, and strongly favors a planetary system.
\end{abstract}

Key words. techniques: radial velocities - planets and satellites: detection - planets and satellites: dynamical evolution and stability planetary systems

\section{Introduction}

Until May 2014, 387 known multiple planet systems were reported in the literature ${ }^{1}$, and their number is constantly growing. The first strong evidence for a multiple planetary system around a main-sequence star was reported by Butler et al. (1999), showing that together with the 4.6 day-period radial velocity signal of $v$ And (Butler et al. 1997), two more long-period, substellar companions can be derived from the Doppler curve. Later, a second Jupiter-mass planet was found to orbit the star $47 \mathrm{UMa}$ (Fischer et al. 2002), and another one the G star HD 12661 (Fischer et al. 2003).

Interesting cases also include planets locked in mean motion resonance (MMR), such as the short-period 2:1 resonance pair around GJ 876 (Marcy et al. 2001), the 3:1 MMR planetary

^ Based on observations collected at Lick Observatory, University of California.

$\star \star$ Based on observations collected at the European Southern Observatory, Chile, under program IDs 088.D-0132, 089.D-0186, 090.D-0155 and 091.D-0365.

1 http://www . exoplanets.org system around HD 60532 (Desort et al. 2008; Laskar \& Correia 2009), or the 3:2 MMR system around HD 45364 (Correia et al. 2009). Follow-up radial velocity observations of well known planetary pairs showed evidence that some of them are actually part of higher-order multiple planetary systems. For example, two additional long-period planets are orbiting around GJ 876 (Rivera et al. 2010), and up to five planets are known to orbit 55 Cnc (Fischer et al. 2008). More recently, Lovis et al. (2011) announced a very dense, but still well-separated lowmass planetary system around the solar-type star HD 10180. Tuomi (2012) claimed that there might even be nine planets in this system, which would make it the most compact and populated extrasolar multiple system known to date.

Now, almost two decades since the announcement of 51 Peg b (Mayor \& Queloz 1995), 55 multiple planetary systems have been found using high-precision Doppler spectroscopy $^{2}$. Another 328 multiple planetary systems have been found with the transiting technique, the vast majority of them with the Kepler satellite. Techniques such as direct

2 http://www . exoplanets.org 
imaging (Marois et al. 2010) and micro-lensing (Gaudi et al. 2008) have also proven to be successful in detecting multiple extrasolar planetary systems.

The different techniques for detecting extrasolar planets and the combination between them shows that planetary systems appear to be very frequent in all kinds of stable configurations. Planetary systems are found to orbit around stars with different ages and spectral classes, including binaries (Lee et al. 2009) and even pulsars (Wolszczan \& Frail 1992). Nevertheless, not many multiple planetary systems have been found around evolved giant stars so far. The multiple planetary systems appear to be a very small fraction of the planet occurrence statistics around evolved giants, which are dominated by single planetary systems. Up to date there is only one multiple planetary system candidate known around an evolved star (HD 102272, Niedzielski et al. 2009a), and two multiple systems consistent with brown dwarf mass companions around BD +20 2457 (Niedzielski et al. 2009b) and $v$ Oph (Quirrenbach et al. 2011; Sato et al. 2012).

In this paper we present evidence for two Jovian planets orbiting the $\mathrm{K}$ giant $\eta$ Cet based on precise radial velocities. We also carry out an extensive stability analysis to demonstrate that the system is stable and to further constrain its parameters.

The outline of the paper is as follows: in Sect. 2 we introduce the stellar parameters for $\eta$ Cet and describe our observations taken at Lick observatory and at the VLT. Section 3 describes the derivation of the spectroscopic orbital parameters. In Sect. 4 we explain our dynamical stability calculations, and in Sect. 5 we discuss the possible origin of the $\eta$ Cet system and the population of planets around giants. Finally, we provide a summary in Sect. 6.

\section{Observations and stellar characteristics}

\subsection{K giant star $\eta$ Cet}

$\eta$ Cet (=HIP 5364, HD 6805, HR 334) is a bright $(V=$ $3.46 \mathrm{mag})$, red giant clump $\operatorname{star}(B-V=1.16)$. It is located at a distance of $37.99 \pm 0.20 \mathrm{pc}$ (van Leeuwen 2007) and flagged in the HIPPARCos catalog as photometrically constant.

Luck \& Challener (1995) proposed $T_{\text {eff }}=4425 \pm$ $100 \mathrm{~K}$, derived from photometry, and $\log g=2.65 \pm$ $0.25\left[\mathrm{~cm} \mathrm{~s}^{-2}\right]$ estimated from the ionization balance between Fe I and Fe II lines in the spectra. Luck \& Challener (1995) derived $[\mathrm{Fe} / \mathrm{H}]=0.16 \pm 0.05$, and a mass of $M=1.3 \pm 0.2 M_{\odot}$. The more recent study of $\eta$ Cet by Berio et al. (2011) derived the stellar parameters as $T_{\text {eff }}=4356 \pm 55 \mathrm{~K}$, luminosity $L=74.0 \pm 3.7 L_{\odot}$, and the estimated radius as $R=15.10 \pm 0.10 R_{\odot}$. Berio et al. (2011) roughly estimate the mass of $\eta$ Cet to be $M=1.0-1.4 M_{\odot}$ by comparing its position in the Hertzsprung-Russell (HR) diagram with evolutionary tracks of solar metallicity.

By using a Lick template spectrum without iodine absorption cell lines, Hekker \& Meléndez (2007) estimated the metallicity of $\eta$ Cet to be $[\mathrm{Fe} / \mathrm{H}]=0.07 \pm 0.1$. Based on this metallicity and the observed position in the HR diagram, a trilinear interpolation in the evolutionary tracks and isochrones (Girardi et al. 2000) yields $T_{\text {eff }}=4529 \pm 19 \mathrm{~K}, \log g=2.36 \pm 0.05\left[\mathrm{~cm} \mathrm{~s}^{-2}\right], L=$ $77.1 \pm 1.1 L_{\odot}$ and $R=14.3 \pm 0.2 R_{\odot}$ (Reffert et al. 2014).

We determined the probability of $\eta$ Cet to be on the red giant branch (RGB) or on the horizontal branch (HB) by generating 10000 positions with $\left(B-V, M_{V},[\mathrm{Fe} / \mathrm{H}]\right)$ consistent with the error bars on these quantities, and derived the stellar parameters via a comparison with interpolated evolutionary tracks. Our method for deriving stellar parameters for all $\mathrm{G}$ and $\mathrm{K}$ giant stars
Table 1. Stellar properties of $\eta$ Cet.

\begin{tabular}{lcl}
\hline \hline Parameter & $\eta$ Cet & Reference \\
\hline Spectral type & K1III & Gray et al. (2006) \\
& K2III CNO.5 & Luck \& Challener (1995) \\
$m_{v}[\mathrm{mag}]$ & 3.46 & van Leeuwen (2007) \\
$B-V$ & $1.161 \pm 0.005$ & van Leeuwen (2007) \\
Distance [pc] & $37.99 \pm 0.20$ & van Leeuwen (2007) \\
$\pi$ [mas] & $26.32 \pm 0.14$ & van Leeuwen (2007) \\
Mass $\left[M_{\odot}\right]$ & $1.7 \pm 0.1$ & Reffert et al. (2014) \\
& $1.3 \pm 0.2$ & Luck \& Challener (1995) \\
& $1.2 \pm 0.2$ & Berio et al. (2011) \\
Luminosity $\left[L_{\odot}\right]$ & $77.1 \pm 1.1$ & Reffert et al. (2014) \\
& $74.0 \pm 3.7$ & Berio et al. (2011) \\
Radius $\left[R_{\odot}\right]$ & $14.3 \pm 0.2$ & Reffert et al. (2014) \\
& $15.1 \pm 0.1$ & Berio et al. (2011) \\
$T_{\text {eff }}[\mathrm{K}]$ & $4528 \pm 19$ & Reffert et al. (2014) \\
& $4563 \pm 82$ & Prugniel et al. (2011) \\
& $4425 \pm 100$ & Luck \& Challener (1995) \\
$\log g\left[\mathrm{~cm} \mathrm{~s}^{-2}\right]$ & $4356 \pm 55$ & Berio et al. (2011) \\
& $2.36 \pm 0.05$ & Reffert et al. (2014) \\
{$[$ Fe/H] } & $2.61 \pm 0.21$ & Prugniel et al. (2011) \\
& $2.65 \pm 0.25$ & Luck \& Challener (1995) \\
$v \sin i\left[\mathrm{~km} \mathrm{~s}^{-1}\right]$ & $0.07 \pm 0.1$ & Hekker \& Meléndez (2007) \\
$R V_{\text {absolute }}\left[\mathrm{km} \mathrm{s}^{-1}\right]$ & $0.12 \pm 0.08$ & Prugniel et al. (2011) \\
\hline & $11.8 \pm 0.6^{\alpha}$ & Luck \& Challener (1995) \\
& & Hekker \& Meléndez (2007) \\
& & This paper \\
\hline
\end{tabular}

Notes. $\alpha$ : we estimated this using $\sigma_{F W H M}$ and $\sigma_{v_{\text {mac }}}$ given in Hekker \& Meléndez (2007).

monitored at Lick Observatory, including $\eta$ Cet, is described in more detail in Reffert et al. (2014).

Our results show that $\eta$ Cet has a $70 \%$ probability to be on the RGB with a resulting mass of $M=1.7 \pm 0.1 M_{\odot}$. If $\eta \mathrm{Cet}$ were on the $\mathrm{HB}$, the mass would be $M=1.6 \pm 0.2 M_{\odot}$. Here we simply use the mass with the highest probability. All stellar parameters are summarized in Table 1.

\subsection{Lick data set}

Doppler measurements for $\eta$ Cet have been obtained since July 2000 as part of our precise $\left(5-8 \mathrm{~m} \mathrm{~s}^{-1}\right)$ Doppler survey of 373 very bright ( $V \leq 6 \mathrm{mag}$ ) $\mathrm{G}$ and $\mathrm{K}$ giants. The program started in June 1999 using the $0.6 \mathrm{~m}$ Coudé Auxiliary Telescope (CAT) with the Hamilton Échelle Spectrograph at Lick Observatory. The original goal of the program was to study the intrinsic radial velocity variability in $\mathrm{K}$ giants, and to demonstrate that the low levels of stellar jitter make these stars a good choice for astrometric reference objects for the Space Interferometry Mission (SIM; Frink et al. 2001). However, the low amplitude of the intrinsic jitter of the selected $\mathrm{K}$ giants, together with the precise and regular observations, makes this survey sensitive to variations in the radial velocity that might be caused by extrasolar planets.

All observations at Lick Observatory have been taken with the iodine cell placed in the light path at the entrance of the spectrograph. This technique provides us with many narrow and very well defined iodine spectral lines, which are used as references, and it is known to yield precise Doppler shifts down to $3 \mathrm{~m} \mathrm{~s}^{-1}$ or even better for dwarf stars (Butler et al. 1996). The iodine method is not discussed in this paper; instead we refer to Marcy \& Butler (1992), Valenti et al. (1995), and Butler et al. (1996), 




Fig. 1. Top panel: radial velocities measured at Lick Observatory (blue circles), along with error bars, covering about 11 years from July 2000 to October 2011. Two best fits to the Lick data are overplotted: a double-Keplerian fit (dot-dashed) and the best dynamical edge-on coplanar fit (solid line). The two fits are not consistent in later epochs because of the gravitational interactions considered in the dynamical model. Despite the large estimated errors, the data from CRIRES (red diamonds) seem to follow the best fit prediction from the dynamical fit. Bottom panel: no systematics are visible in the residuals. The remaining radial velocity scatter has a standard deviation of $15.9 \mathrm{~m} \mathrm{~s}^{-1}$, most likely caused by rapid solar - like $p$ - mode oscillations.

where more details about the technique and the data reduction can be found.

The wavelength coverage of the Hamilton spectra extends from 3755 to $9590 \AA$, with a resolution of $R \approx 60000$ in the wavelength range from 5000 to $5800 \AA$, where most of the iodine lines can be found and where the radial velocities are measured. The typical exposure time with the $0.6 \mathrm{~m} \mathrm{CAT}$ is $450 \mathrm{~s}$, which results in a signal-to-noise ratio $(\mathrm{S} / \mathrm{N})$ of about 100 , reaching a radial velocity precision of better than $5 \mathrm{~m} \mathrm{~s}^{-1}$. The individual radial velocities are listed in Table 2, together with Julian dates and their formal errors.

\subsection{CRIRES data set}

Nine additional Doppler measurements for $\eta$ Cet were taken between October 2011 and July 2013 with the pre-dispersed CRyogenic InfraRed Echelle Spectrograph (CRIRES) mounted at VLT UT1 (Antu), (Kaeufl et al. 2004). CRIRES has a resolving power of $R \approx 100000$ when used with a $0.2^{\prime \prime}$ slit, covering a narrow wavelength region in the $J, H, K, L$ or $M$ infrared bands (960-5200 nm). Several studies have demonstrated that radial velocity measurements with precision between 10 and $35 \mathrm{~m} \mathrm{~s}^{-1}$ are possible with CRIRES. Seifahrt \& Käufl (2008) reached a precision of $\approx 35 \mathrm{~m} \mathrm{~s}^{-1}$ when using reference spectra of a $\mathrm{N}_{2} \mathrm{O}$ gas cell, and Bean \& Seifahrt (2009) even reached $\approx 10 \mathrm{~m} \mathrm{~s}^{-1}$ with an ammonia $\left(\mathrm{NH}_{3}\right)$ gas-cell. Huélamo et al. (2008) and Figueira et al. (2010) showed that the achieved Doppler precision can be better than $\approx 25 \mathrm{~m} \mathrm{~s}^{-1}$ when using telluric absorption lines in the $H$ band as reference spectra.

Motivated by these results, our strategy with CRIRES is to test the optical Doppler data with those from the near-IR regime for the objects in our $\mathrm{G}$ and $\mathrm{K}$ giants sample that clearly exhibit a



Fig. 2. Top panel: the periodogram of the measured radial velocities shows two highly significant peaks around 399 and 768 days, while the Kepler fit to the data reveals a best fit at periods around 403.5 and 751.9 days. Bottom panel: no significant peak is left in the periodogram of the residuals after removing the two periods from the Keplerian fit.

periodicity consistent with one or more substellar companion(s). If the periodic Doppler signal were indeed caused by a planet, we would expect the near-IR radial velocities to follow the best-fit model derived from the optical spectra.

If the radial velocity variations in the optical and in the nearIR are not consistent, the reason may be either large stellar spots (Huélamo et al. 2008; Bean et al. 2010) or nonradial pulsations that will result in a different velocity amplitude at visible and infrared wavelengths (Percy et al. 2001). When stellar spots mimic a planetary signal, the contrast between the flux coming from the stellar photosphere and the flux coming from the cooler spot is higher at optical wavelengths and thus has a higher RV amplitude than the near-IR.

For our observation with CRIRES we decided to adopt an observational setup similar to that successfully used by Figueira et al. (2010). We chose a wavelength setting in the $H$ band $(36 / 1 / \mathrm{n})$, with a reference wavelength of $\lambda_{\text {ref }}=1594.5 \mathrm{~nm}$. This particular region was selected by inspecting the Arcturus near-IR spectral atlas from Hinkle et al. (1995) and searching for a good number of stellar as well as telluric lines. The selected spectral region is characterized by many deep and sharp atmospheric $\mathrm{CO}_{2}$ lines that take the role of an always available on-sky gas cell. To achieve the highest possible precision the spectrograph was used with a resolution of $R=100000$. To avoid RV errors related to a nonuniform illumination of the slit, the observations were made in NoAO mode (without adaptive optics), and nights with poor seeing conditions were requested.

Values for the central wavelengths of the telluric $\mathrm{CO}_{2}$ lines were obtained from the HITRAN database (Rothman et al. 1998), allowing us to construct an accurate wavelength solution for each detector frame. The wavelengths of the identified stellar spectral lines were taken from the Vienna Atomic Line Database (VALD; Kupka et al. 1999), based on the target's $T_{\text {eff }}$ and $\log g$.

Dark, flat, and nonlinearity corrections and the combination of the raw jittered frames in each nodding position were performed using the standard ESO CRIRES pipeline recipes. Later, the precise RV was obtained from a cross-correlation (Baranne et al. 1996) of the science spectra and the synthetic telluric and 
Table 2. Measured velocities for $\eta$ Cet and derived errors.

\begin{tabular}{crc}
\hline \hline $\mathrm{JD}$ & $R V\left[\mathrm{~m} \mathrm{~s}^{-1}\right]$ & $\sigma_{\mathrm{RV}}\left[\mathrm{m} \mathrm{s}^{-1}\right]$ \\
\hline 2451745.994 & 37.8 & 4.3 \\
2451778.895 & 24.7 & 3.7 \\
2451808.867 & -12.2 & 3.6 \\
2451853.766 & -2.1 & 4.1 \\
2451856.801 & 0.1 & 4.6 \\
2451898.637 & -4.0 & 8.0 \\
2451932.609 & 23.9 & 3.9 \\
2452163.861 & -89.3 & 4.9 \\
2452175.851 & -75.7 & 4.0 \\
2452307.598 & -98.1 & 4.5 \\
2452465.989 & 96.1 & 5.3 \\
2452483.985 & 81.6 & 5.0 \\
2452494.987 & 66.0 & 5.0 \\
2452517.936 & 35.5 & 5.2 \\
2452528.910 & 52.4 & 5.2 \\
2452530.910 & 43.7 & 4.5 \\
2452541.904 & 45.1 & 4.5 \\
2452543.883 & 48.3 & 5.0 \\
2452559.914 & 30.5 & 6.4 \\
2452561.795 & 14.0 & 8.4 \\
2452571.791 & 29.7 & 5.0 \\
2452589.810 & 18.3 & 5.7 \\
2452603.782 & 10.4 & 4.9 \\
2452605.724 & 22.9 & 4.8 \\
2452616.772 & 19.9 & 5.9 \\
2452665.604 & 8.5 & 4.2 \\
2452668.611 & -8.0 & 5.3 \\
2452837.972 & 31.3 & 5.4 \\
2452861.963 & -4.6 & 4.1 \\
2452863.985 & 19.0 & 5.2 \\
2452879.918 & -0.9 & 4.2 \\
2452880.947 & 1.3 & 5.0 \\
2452898.911 & 6.1 & 4.7 \\
2452900.928 & 11.4 & 4.8 \\
2452932.839 & -6.3 & 4.3 \\
2452934.804 & -29.9 & 4.6 \\
2452963.753 & -41.5 & 5.2 \\
2452965.809 & -11.9 & 5.4 \\
2453022.630 & -95.6 & 5.6 \\
2453208.010 & 22.7 & 3.8 \\
2453209.978 & 32.5 & 3.7 \\
2453214.961 & 35.2 & 4.2 \\
& & \\
\hline & &
\end{tabular}

\begin{tabular}{crc}
\hline \hline JD & $R V\left[\mathrm{~m} \mathrm{~s}^{-1}\right]$ & $\sigma_{\mathrm{RV}}\left[\mathrm{m} \mathrm{s}^{-1}\right]$ \\
\hline 2453231.961 & 64.3 & 4.0 \\
2453233.909 & 69.9 & 3.9 \\
2453235.960 & 62.6 & 4.2 \\
2453265.899 & 74.7 & 3.8 \\
2453268.919 & 93.4 & 4.0 \\
2453269.810 & 80.0 & 3.5 \\
2453286.828 & 73.1 & 3.8 \\
2453324.713 & 80.0 & 5.5 \\
2453354.654 & 50.9 & 3.8 \\
2453400.642 & 59.6 & 7.5 \\
2453550.983 & -14.9 & 4.4 \\
2453578.964 & 12.6 & 4.6 \\
2453613.923 & 6.0 & 4.2 \\
2453654.771 & -4.9 & 4.1 \\
2453701.724 & -26.0 & 4.6 \\
2453740.745 & -20.1 & 4.7 \\
2453911.993 & -33.7 & 3.6 \\
2453917.013 & -49.9 & 3.9 \\
2453934.974 & -28.0 & 3.9 \\
2453968.925 & 9.9 & 3.2 \\
2453981.851 & 21.6 & 3.8 \\
2453985.899 & 55.2 & 4.0 \\
2454055.755 & 56.8 & 3.9 \\
2454297.975 & -60.6 & 4.1 \\
2454300.974 & -73.2 & 4.0 \\
2454314.939 & -86.2 & 3.6 \\
2454318.981 & -69.2 & 4.2 \\
2454344.859 & -80.2 & 4.3 \\
2454348.917 & -75.8 & 4.0 \\
2454391.887 & -31.6 & 5.4 \\
2454394.775 & -32.4 & 4.2 \\
2454418.777 & 1.2 & 4.5 \\
2454420.763 & 2.7 & 4.2 \\
2454440.653 & -10.6 & 3.4 \\
2454443.651 & -26.0 & 3.6 \\
2454481.638 & 1.2 & 3.9 \\
2454503.651 & -9.5 & 4.3 \\
2454645.984 & -44.1 & 4.5 \\
2454646.968 & -46.2 & 4.2 \\
2454668.009 & -63.5 & 3.7 \\
2454681.904 & -31.4 & 3.8 \\
2454683.970 & -47.0 & 3.8 \\
& & \\
\hline
\end{tabular}

\begin{tabular}{crc}
\hline \hline JD & $R V\left[\mathrm{~m} \mathrm{~s}^{-1}\right]$ & $\sigma_{\mathrm{RV}}\left[\mathrm{m} \mathrm{s}^{-1}\right]$ \\
\hline 2454711.893 & -32.4 & 4.1 \\
2454713.873 & -31.0 & 4.2 \\
2454715.882 & -17.4 & 4.6 \\
2454716.875 & -24.0 & 4.1 \\
2454753.885 & -3.2 & 3.6 \\
2454755.850 & 6.4 & 3.8 \\
2454777.733 & 41.3 & 4.1 \\
2454806.796 & 94.7 & 5.5 \\
2454808.770 & 90.8 & 4.3 \\
2454809.763 & 113.7 & 6.1 \\
2455026.973 & -30.0 & 5.7 \\
2455063.965 & -43.9 & 5.0 \\
2455099.996 & -52.8 & 4.9 \\
2455116.856 & -63.9 & 6.8 \\
2455154.739 & -82.1 & 7.6 \\
2455174.788 & -89.3 & 5.8 \\
2455364.972 & -11.9 & 5.0 \\
2455419.012 & -29.9 & 4.7 \\
2455446.847 & -35.4 & 3.6 \\
2455446.856 & -36.2 & 3.1 \\
2455463.833 & -59.8 & 5.2 \\
2455466.842 & -33.0 & 4.8 \\
2455569.653 & 40.0 & 4.1 \\
2455571.713 & 59.6 & 4.6 \\
2455572.671 & 65.6 & 4.8 \\
2455621.621 & 121.2 & 4.6 \\
2455757.974 & 39.1 & 5.0 \\
2455803.919 & -5.4 & 4.3 \\
2455806.936 & 3.4 & 4.3 \\
2455828.781 & -30.0 & 4.2 \\
2455831.849 & -24.8 & 5.1 \\
$\star 2455853.844$ & -96.7 & 40.0 \\
$\star 2455854.616$ & -82.0 & 40.0 \\
2455861.842 & -57.5 & 4.2 \\
2455864.796 & -59.9 & 4.4 \\
2455892.745 & -69.9 & 4.9 \\
$\star 2456113.863$ & -60.0 & 40.0 \\
$\star 2456121.811$ & 9.1 & 40.0 \\
$\star 2456139.747$ & -36.2 & 40.0 \\
$\star 2456239.563$ & -54.2 & 40.0 \\
2456411.910 & 77.7 & 40.0 \\
2456499.917 & 47.9 & 40.0 \\
\hline & 72.4 & 40.0 \\
\hline
\end{tabular}

Notes. ${ }^{(\star)}$ CRIRES data.

stellar mask; this was obtained for each frame and nodding position individually. We estimate the formal error of our CRIRES measurements to be on the order of $\sim 40 \mathrm{~m} \mathrm{~s}^{-1}$, based on the rms dispersion values around the best fits for all good targets of our CRIRES sample. This error is probably overestimated, in particular, for the $\eta$ Cet data set.

The full procedure of radial velocity extraction based on the cross-correlation method will be described in more detail in a follow-up paper (Trifonov et al., in prep.).

The CRIRES observations of $\eta$ Cet were taken with an exposure time of $3 \mathrm{~s}$, resulting in a $\mathrm{S} / \mathrm{N}$ of $\approx 300$. Our measured CRIRES radial velocities for $\eta$ Cet are shown together with the data from Lick Observatory in Fig. 1, while the measured values are given in Table 2 .

\section{Orbital fit}

Our measurements for $\eta$ Cet, together with the formal errors and the best Keplerian and dynamical edge-on coplanar fits to the data, are shown in Fig. 1. We used the Systemic Console package (Meschiari et al. 2009) for the fitting.

A preliminary test for periodicities with a Lomb-Scargle periodogram shows two highly significant peaks around 399 and 768 days, suggesting two substellar companions around $\eta$ Cet (see Fig. 2).

The sum of two Keplerian orbits provides a reasonable explanation of the $\eta$ Cet radial velocity data (see Fig. 1). However, the relatively close planetary orbits and their derived minimum masses raise the question whether this planetary system suffers from sufficient gravitational perturbations between the bodies that might be detected in the observed data. For this reason we decided to use Newtonian dynamical fits, applying the Gragg-Bulirsch-Stoer integration method (B-SM: Press et al. 1992), built into Systemic. In this case gravitational perturbations that occur between the planets are taken into account in the model.

We used the simulated annealing method (SA: Press et al. 1992) to determine whether there is more than one $\chi_{\text {red }}^{2}$ local minimum in the data. When the global minimum is found, the derived Jacobi orbital elements from the dynamical fit are the masses of the planets $m_{b, c}$, the periods $P_{b, c}$, eccentricities $e_{b, c}$, longitudes of periastron $\varpi_{b, c}$, and the mean anomaly $M_{b, c}(b$ always denotes the inner planet and $c$ the outer planet). To explore the statistical and dynamical properties of the fits around the best fit, we adopted the systematic grid-search techniques coupled with dynamical fitting. This technique is fully described for the HD 82943 two-planet system (Tan et al. 2013). 
T. Trifonov et al.: Precise radial velocities of giant stars. VI.

Table 3. $\eta$ Cet system best fits (Jacobi coordinates).

\begin{tabular}{lcc}
\hline \hline Orb. Param. & $\eta$ Cet b & $\eta$ Cet c \\
\hline Best Keplerian & & \\
\hline$P[$ days $]$ & $403.5 \pm 1.5$ & $751.9 \pm 3.8$ \\
$m\left[M_{\text {Jup }}\right]$ & $2.55 \pm 0.13$ & $3.32 \pm 0.18$ \\
$e$ & $0.13 \pm 0.05$ & $0.1 \pm 0.06$ \\
$M[\mathrm{deg}]$ & $193.5 \pm 24.6$ & $240.5 \pm 34.8$ \\
$\varpi[\mathrm{deg}]$ & $250.6 \pm 20.5$ & $67.54 \pm 5.2$ \\
$K_{1}\left[\mathrm{~m} \mathrm{~s}^{-1}\right]$ & 49.7 & 52.4 \\
$a[\mathrm{AU}]$ & 1.27 & 1.93 \\
$\mathrm{rms}\left[\mathrm{m} \mathrm{s}^{-1}\right]$ & 15.9 & \\
$R V_{\text {offset }}\left[\mathrm{m} \mathrm{s}^{-1}\right]$ & -0.77 & \\
$\chi_{\text {red }}$ & $13.67(1.17$ with jitter $)$ \\
\hline
\end{tabular}

\begin{tabular}{|c|c|c|}
\hline Orb. Param. & $\eta$ Cet b & $\eta$ Cet c \\
\hline \multicolumn{3}{|c|}{ Best coplanar edge-on } \\
\hline$P$ [days] & $407.5 \pm 2.67$ & $739.9 \pm 4.8$ \\
\hline$m\left[M_{\mathrm{Jup}}\right]$ & $2.55 \pm 0.16$ & $3.26 \pm 0.17$ \\
\hline$e$ & $0.12 \pm 0.05$ & $0.08 \pm 0.04$ \\
\hline$M[\mathrm{deg}]$ & $208.2 \pm 13.7$ & $227.8 \pm 19.6$ \\
\hline$\varpi[\mathrm{deg}]$ & $245.4 \pm 9.5$ & $68.2 \pm 22.3$ \\
\hline$K_{1}\left[\mathrm{~m} \mathrm{~s}^{-1}\right]$ & 49.4 & 51.6 \\
\hline$a[\mathrm{AU}]$ & 1.28 & 1.91 \\
\hline $\operatorname{rms}\left[\mathrm{m} \mathrm{s}^{-1}\right]$ & 15.19 & \\
\hline$R V_{\text {offset }}\left[\mathrm{m} \mathrm{s}^{-1}\right]$ & 0.0 & \\
\hline$\chi_{\text {red }}^{2}$ & 11.39 (1.001 with jitter) & \\
\hline \multicolumn{3}{|c|}{ Best coplanar inclined } \\
\hline$P$ [days] & $396.8 \pm 0.1$ & $767.1 \pm 0.27$ \\
\hline$m\left[M_{\mathrm{Jup}}\right]$ & $3.85 \pm 0.03$ & $5.52 \pm 0.02$ \\
\hline$e$ & $0.24 \pm 0.05$ & $0.1 \pm 0.01$ \\
\hline$M[\mathrm{deg}]$ & $163.3 \pm 0.1$ & $78.8 \pm 0.05$ \\
\hline$\varpi[\mathrm{deg}]$ & $292.2 \pm 0.1$ & $221.3 \pm 0.1$ \\
\hline$i[\mathrm{deg}]$ & 35.5 & 35.5 \\
\hline$K_{1}\left[\mathrm{~m} \mathrm{~s}^{-1}\right]$ & 44.8 & 50.2 \\
\hline$a[\mathrm{AU}]$ & 1.26 & 1.96 \\
\hline $\operatorname{rms}\left[\mathrm{m} \mathrm{s}^{-1}\right]$ & 14.56 & \\
\hline$R V_{\text {offset }}\left[\mathrm{m} \mathrm{s}^{-1}\right]$ & -3.28 & \\
\hline$\chi_{\mathrm{red}}^{2}$ & 10.89 (0.925 with jitter) & \\
\hline \multicolumn{3}{|c|}{ Best mutually inclined } \\
\hline$P$ [days] & $404.4 \pm 2.7$ & $748.2 \pm 6.8$ \\
\hline$m\left[M_{\mathrm{Jup}}\right]$ & $5.5 \pm 1.08$ & $7.74 \pm 1.45$ \\
\hline$e$ & $0.06 \pm 0.06$ & $0.05 \pm 0.07$ \\
\hline$M[\mathrm{deg}]$ & $168.4 \pm 28.1$ & $145.3 \pm 31.1$ \\
\hline$\varpi[\mathrm{deg}]$ & $283.7 \pm 23.3$ & $138.8 \pm 70.2$ \\
\hline$i[\mathrm{deg}]$ & $151.7 \pm 24.9$ & $155.0 \pm 34.6$ \\
\hline$\Delta \Omega[\mathrm{deg}]$ & $345.4 \pm 46.4$ & \\
\hline$K_{1}\left[\mathrm{~m} \mathrm{~s}^{-1}\right]$ & 50.2 & 52.1 \\
\hline$a[\mathrm{AU}]$ & 1.28 & 1.93 \\
\hline $\mathrm{rms}\left[\mathrm{m} \mathrm{s}^{-1}\right]$ & 14.61 & \\
\hline$R V_{\text {offset }}\left[\mathrm{m} \mathrm{s}^{-1}\right]$ & -1.89 & \\
\hline$\chi_{\text {red }}^{2}$ & 10.90 (0.95 with jitter) & \\
\hline
\end{tabular}

\begin{tabular}{|c|c|c|}
\hline Orb. Param. & $\eta$ Cet b & $\eta$ Cet c \\
\hline \multicolumn{3}{|c|}{ 2:1 MMR coplanar edge-on } \\
\hline$P$ [days] & $407.5 \pm 2.67$ & $744.5 \pm 3.71$ \\
\hline$m\left[M_{\text {Jup }}\right]$ & $2.54 \pm 0.16$ & $3.28 \pm 0.19$ \\
\hline$e$ & $0.155 \pm 0.05$ & $0.025 \pm 0.05$ \\
\hline$M[\mathrm{deg}]$ & $211.1 \pm 45.33$ & $268.0 \pm 21.33$ \\
\hline$\varpi[\mathrm{deg}]$ & $244.7 \pm 31.64$ & $32.5 \pm 32.72$ \\
\hline$K_{1}\left[\mathrm{~m} \mathrm{~s}^{-1}\right]$ & 49.6 & 51.7 \\
\hline$a[\mathrm{AU}]$ & 1.28 & 1.92 \\
\hline $\mathrm{rms}\left[\mathrm{m} \mathrm{s}^{-1}\right]$ & 15.28 & \\
\hline$R V_{\text {offset }}\left[\mathrm{m} \mathrm{s}^{-1}\right]$ & -0.08 & \\
\hline$\chi_{\text {red }}^{2}$ & 11.65 (1.013 with jitter) & \\
\hline \multicolumn{3}{|c|}{ 2:1 MMR coplanar inclined } \\
\hline$P$ [days] & $407.3 \pm 2.1$ & $744.3 \pm 4.1$ \\
\hline$m\left[M_{\text {Jup }}\right]$ & $2.46 \pm 0.12$ & $3.16 \pm 0.2$ \\
\hline$e$ & $0.17 \pm 0.05$ & $0.02 \pm 0.03$ \\
\hline$M[\mathrm{deg}]$ & $208.9 \pm 16.1$ & $262.7 \pm 41.2$ \\
\hline$\varpi[\mathrm{deg}]$ & $247.2 \pm 13.5$ & $36.67 \pm 41.1$ \\
\hline$i[\mathrm{deg}]$ & 81.9 & 81.9 \\
\hline$K_{1}\left[\mathrm{~m} \mathrm{~s}^{-1}\right]$ & 49.8 & 51.6 \\
\hline$a[\mathrm{AU}]$ & 1.28 & 1.92 \\
\hline $\operatorname{rms}\left[\mathrm{m} \mathrm{s}^{-1}\right]$ & 15.2 & \\
\hline$R V_{\text {offset }}\left[\mathrm{m} \mathrm{s}^{-1}\right]$ & -0.58 & \\
\hline$\chi_{\text {red }}^{2}$ & 11.84 (1.04 with jitter) & \\
\hline \multicolumn{3}{|c|}{ 2:1 MMR mutually inclined } \\
\hline$P$ [days] & $407.8 \pm 2.9$ & $742.2 \pm 4.5$ \\
\hline$m\left[M_{\text {Jup }}\right]$ & $2.45 \pm 0.12$ & $3.14 \pm 0.17$ \\
\hline$e$ & $0.13 \pm 0.05$ & $0.06 \pm 0.04$ \\
\hline$M[\mathrm{deg}]$ & $209.0 \pm 15.4$ & $247.2 \pm 54.5$ \\
\hline$\varpi[\mathrm{deg}]$ & $246.8 \pm 12.7$ & $51.1 \pm 52.2$ \\
\hline$i[\mathrm{deg}]$ & 88.0 & 92.0 \\
\hline$\Delta \Omega[\mathrm{deg}]$ & 0.0 & \\
\hline$K_{1}\left[\mathrm{~m} \mathrm{~s}^{-1}\right]$ & 47.7 & 49.5 \\
\hline$a[\mathrm{AU}]$ & 1.29 & 1.92 \\
\hline $\operatorname{rms}\left[\mathrm{m} \mathrm{s}^{-1}\right]$ & 15.4 & \\
\hline$R V_{\text {offset }}\left[\mathrm{m} \mathrm{s}^{-1}\right]$ & -0.46 & \\
\hline$\chi_{\text {red }}^{2}$ & 11.84 (1.03 with jitter) & \\
\hline
\end{tabular}

It is important to note that a good fit means that the $\chi_{\text {red }}^{2}$ solution is close to one. In our case the best edge-on coplanar fit has $\chi_{\text {red }}^{2}=11.39$ (see Sect. 3.1) for 118 radial velocity data points, meaning that the data are scattered around the fit, and this can indeed be seen in Fig. 1. The reason for that is additional radial velocity stellar jitter of about $\sim 15 \mathrm{~m} \mathrm{~s}^{-1}$ that is not taken into account in the weights of each data point.

This jitter value was determined directly as the rms of the residual deviation around the model. In fact, this value is close to the bottom envelope of the points in Fig. 3 for $\eta$ Cet's color index $(B-V=1.16)$, which is likely the lowest jitter for $\eta$ Cet. Based on the long period of our study and the large sample of stars with similar physical characteristics, we found that the intrinsic stellar jitter is clearly correlated with the color index $B-V$ of the stars. and its value for $\eta$ Cet is typical for other K2 III giants in our Lick sample (see Fig. 3). For $\eta$ Cet we estimated an

expected jitter value of $25 \mathrm{~m} \mathrm{~s}^{-1}$ (see Fig. 3), which is higher than the jitter estimated from the rms of the fit. It is also known that late G giants (Frandsen et al. 2002; De Ridder et al. 2006) and K giants (Barban et al. 2004; Zechmeister et al. 2008) exhibit rapid solar-like $p$-mode oscillations, much more rapid than the typical time sampling of our observations, which appear as scatter in our data. Using the scaling relation from Kjeldsen \& Bedding $(1995,2011)$, the stellar oscillations for $\eta$ Cet are estimated to have a period of $\sim 0.4$ days and an amplitude of $\sim 11 \mathrm{~m} \mathrm{~s}^{-1}$, which again agrees well with $\eta$ Cet's RV scatter level around the fit.

We re-assessed the $\chi_{\text {red }}^{2}$ by quadratically adding the stellar jitter to the formal observational errors $\left(3-5 \mathrm{~m} \mathrm{~s}^{-1}\right.$ ) for each radial velocity data point, which scaled down the $\chi_{\text {red }}^{2}$ of the best fit close to unity. For our edge-on coplanar case $\chi_{\text {red }}^{2}=11.39$ 


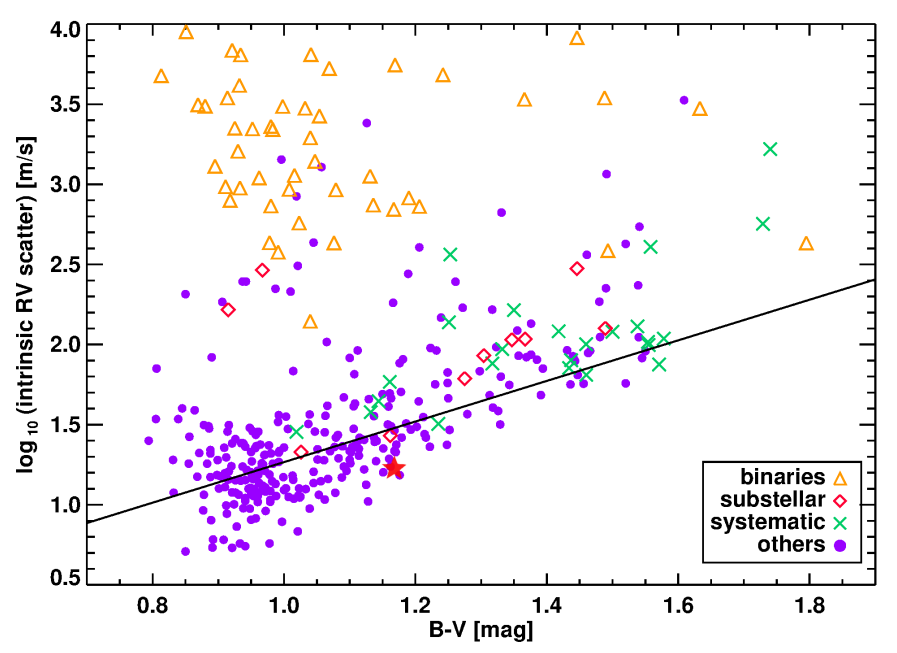

Fig. 3. Intrinsic RV scatter observed in our sample of $373 \mathrm{~K}$ giants versus $B-V$ color. A clear trend is visible in the sense that redder stars without companions (circles) have larger intrinsic RV variations. A number of stars lie above the almost linear relation between color and the logarithm of the scatter. These stars have clearly periodic RVs, which indicates that they harbor substellar or stellar companions. Stars with non-periodic, but still systematic radial velocities are indicated with green crosses. The RV scatter of $\sim 15 \mathrm{~m} \mathrm{~s}^{-1}$, for $\eta$ Cet (red star) derived as the rms around the orbital fit, is lower than the $25 \mathrm{~m} \mathrm{~s}^{-1}$ derived from the linear trend at the star's color index.

is scaled down to $\chi_{\text {red }}^{2}=1.001$. We provide both: the unscaled $\chi_{\text {red }}^{2}$ value together with the derived stellar jitter and the $\chi_{\text {red }}^{2}$ value where the average stellar jitter derived above is taken into account.

We estimated the error of the derived orbital parameters using two independent methods available as part of the Console: bootstrap synthetic data-refitting and MCMC statistics, which runs multiple MCMC chains in parallel with an adaptive step length. Both estimators gave similar formal errors for the orbital parameters. However, the MCMC statistics has been proven to provide better estimates of planetary orbit uncertainties than the more robust bootstrap algorithm (e.g. Ford 2005). Therefore, we will use only the MCMC results in this paper.

The nine near-IR Doppler points from CRIRES are overplotted in Fig. 1, but were not used for fitting. We did not consider the CRIRES data because of their large uncertainties and the negligible total weight to the fit, compared with the Lick data. Another complication is the radial velocity offset between the two data sets, which introduces an additional parameter in the $\chi^{2}$ fitting procedure. Nevertheless, the CRIRES data points agree well with the Newtonian fit predictions based on the optical data (see Fig. 1), providing strong evidence for the two-planet hypothesis.

\subsection{Formally best edge-on coplanar fit}

Assuming an edge-on, co-planar planetary system $\left(i_{b, c}=90^{\circ}\right)$, the global minimum has $\chi_{\text {red }}^{2}=11.39$ (1.001 with jitter), which constitutes a significant improvement from the best twoKeplerian fit with $\chi_{\text {red }}^{2}=13.67$ (1.17 with jitter). This $\chi_{\text {red }}^{2}$ improvement indicates that the strong interaction between the two planetary companions is visible in the radial velocity signal even on short timescales. The derived planetary masses are $m_{b} \sin i_{b}=2.5 M_{\text {Jup }}$ and $m_{c} \sin i_{c}=3.3 M_{\text {Jup }}$, with periods of $P_{b}=407.5$ days and $P_{c}=739.9$ days. The eccentricities are moderate $\left(e_{b}=0.12\right.$ and $\left.e_{c}=0.08\right)$, and the longitudes of periastron suggest an anti-aligned configuration with $\varpi_{b}=245.1^{\circ}$ and $\varpi_{c}=68.2^{\circ}$, that is, $\varpi_{b}-\varpi_{c} \approx 180^{\circ}$. Orbital parameters for both planets, together with their formal uncertainties, are summarized in Table 3.

Dynamical simulations, however, indicate that this fit is stable only for $\lesssim 17000 \mathrm{yr}$. After the start of the integrations, the planetary semi-major axes evolution shows a very high perturbation rate with a constant amplitude. Although the initially derived periods do not suggest any low-order MMR, the average planetary periods appear to be in a ratio of $2: 1$ during the first 17000 years of orbital evolution, before the system becomes chaotic and eventually ejects the outer companion. This indicates that within the orbital parameter errors, the system might be in a long-term stable 2:1 MMR. Such stable edge-on cases are discussed in Sects. 4.2 and 4.3. The evolution of the planetary semimajor axes for this best-fit configuration is illustrated in Fig. 4.

\subsection{Formally best inclined fits}

We also tested whether our best dynamical fit improved significantly by allowing the inclinations with respect to the observer's line of sight (LOS), and the longitudes of the ascending nodes of the planets to be $i_{b, c} \neq 90^{\circ}$ and $\Delta \Omega_{b, c}=\Omega_{b}-\Omega_{c} \neq 0$, respectively.

The impact of the LOS inclinations on the fits mainly manifests itself through the derived planetary masses. The mass function is given by

$\frac{\left(m_{\mathrm{p}} \sin i\right)^{3}}{\left(M_{\star}+m_{\mathrm{p}}\right)^{2}}=\frac{P}{2 \pi G} K_{\star}^{3} \sqrt{\left(1-e^{2}\right)^{3}}$,

where $m_{\mathrm{p}}$ is the planetary mass, $M_{\star}$ the stellar mass, and $G$ is the universal gravitational constant, while the other parameters come from the orbital model: period $(P)$, eccentricity $(e)$, and radial velocity amplitude $\left(K_{\star}\right)$. It is easy to see that if we take $\sin i \neq 1\left(i \neq 90^{\circ}\right)$, the mass of the planet must increase to satisfy the right side of the equation. However, we note that this general expression is valid only for the simple case of one planet orbiting a star. Hierarchical two-planet systems and the dependence of the minimum planetary mass on the inclination are better described in Jacobian coordinates. For more details, we refer to the formalism given in Lee \& Peale (2002).

We separated the inclined fits in two different sets, depending on whether the planets are strictly in a coplanar configuration (yet inclined with respect to the LOS), or if an additional mutual inclination angle between the planetary orbits is allowed (i.e., $i_{b}-i_{c} \neq 0^{\circ}$ and $\Omega_{b}-\Omega_{c} \neq 0^{\circ}$ ).

For the inclined co-planar test we set $i_{b}=i_{c}$, but $\sin i_{b, c} \leq 1$, and we fixed the longitudes of ascending nodes to $\Omega_{b}=\Omega_{c}=0^{\circ}$. In the second test the inclinations of both planets were fitted as independent parameters, allowing mutually inclined orbits. However, $i_{b}$ and $i_{c}$ were restricted to not exceed the $\sin i_{b, c}=$ $0.42\left(i_{b, c}=90^{\circ} \pm 65^{\circ}\right)$ limit, where the planetary masses will become very large. Moreover, as discussed in Laughlin et al. (2002) and in Bean \& Seifahrt (2009), the mutual inclination $\left(\Phi_{b, c}\right)$ of two orbits depends not only on the inclinations $i_{b}$ and $i_{c}$, but also on the longitudes of the ascending nodes $\Omega_{b}$ and $\Omega_{c}$ :

$\cos \Phi_{b, c}=\cos i_{b} \cos i_{c}+\sin i_{b} \sin i_{c} \cos \left(\Omega_{b}-\Omega_{c}\right)$.

The longitudes of the ascending node, $\Omega_{b}$ and $\Omega_{c}$, are not restricted and thus can vary in the range from 0 to $2 \pi$. The broad 
T. Trifonov et al.: Precise radial velocities of giant stars. VI.
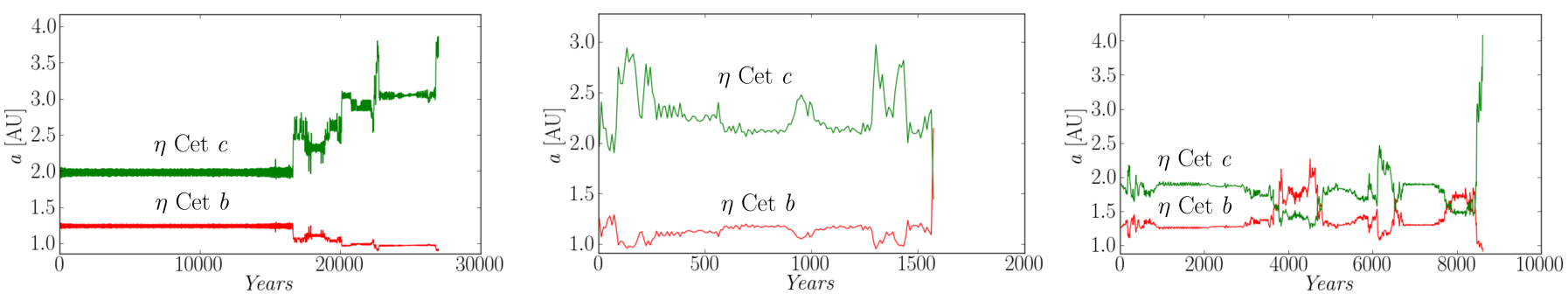

Fig. 4. Semi-major axes evolution of the best dynamical fits. Left: the edge-on coplanar fit remains stable in a 2:1 MMR only for 17000 years, when the system starts to show chaotic behavior and eventually ejects the outer planet. The best inclined coplanar (middle) and mutually inclined (right) fits fail to preserve stability even on very short timescales.

range of $i_{b, c}$ and $\Omega_{b, c}$ might lead to very high mutual inclinations, but in general $\Phi_{b, c}$ was restricted to $50^{\circ}$, although this limit was never reached by the fitting algorithm.

For the coplanar inclined case, the minimum appears to be $\chi_{\text {red }}^{2}=10.89$, while adding the same stellar jitter as above to the data used for the coplanar fit gives $\chi_{\text {red }}^{2}=0.925$. Both planets have orbits with relatively high inclinations with respect to the $\operatorname{LOS}\left(i_{b, c}=35.5^{\circ}\right)$. The planetary masses are $m_{b}=3.85 M_{\text {Jup }}$ and $m_{c}=5.52 M_{\mathrm{Jup}}$, and the planetary periods are closer to the 2:1 ratio: $P_{b}=396.8$ days and $P_{c}=767.1$ days.

The derived mutually inclined best fit has $\chi_{\text {red }}^{2}=10.90$, $\left(\chi_{\text {red }}^{2}=0.95\right)$. This fit also has high planetary inclinations and thus the planetary masses are much more massive: $m_{b}=$ $5.50 M_{\mathrm{Jup}}$ and $m_{c}=7.74 \mathrm{M}_{\mathrm{Jup}}$, while the periods are $P_{b}=$ 404.4 days and $P_{c}=748.2$ days. Orbital parameters and the associated errors for the inclined fits are summarized in Table 3. An F-test shows that the probability that the three additional fitting parameters significantly improve the model is $\sim 90 \%$.

Dynamical simulations based on the inclined fits show that these solutions cannot even preserve stability on very short timescales. The large planetary masses in those cases and the higher interaction rate make these systems much more fragile than the edge-on coplanar system. The best inclined co-planar fit appears to be very unstable and leads to planetary collision in less than 1600 years. The best mutually inclined fit is chaotic from the very beginning of the integrations. During the simulations the planets exchange their positions in the system until the outer planet is ejected after $\sim 9000$ years. The semi-major axes evolution for those systems is illustrated in Fig. 4.

\section{Stability tests}

\subsection{Numerical setup}

For testing the stability of the $\eta$ Cet planetary system we used the Mercury N-body simulator (Chambers 1999), and the SyMBA integrator (Duncan et al. 1998). Both packages have been designed to calculate the orbital evolution of objects moving in the gravitational field of a much more massive central body, as in the case of extrasolar planetary systems. We used Mercury as our primary program and $S y M B A$ to double-check the obtained results. All dynamical simulations were run using the hybrid sympletic/Bulirsch-Stoer algorithm, which is able to compute close encounters between the planets if they occur during the orbital evolution. The orbital parameters for the integrations are taken directly from high-density $\chi_{\text {red }}^{2}$ grids (see Sects. 4.2, 4.3, 4.4 and 4.5$)(\sim 120000$ fits $)$. Our goal is to check the permitted stability regions for the $\eta$ Cet planetary system and to constrain the orbital parameters by requiring stability.

The orbital parameter input for the integrations are in astrocentric format: mean anomaly $M$, semi-major axis $a$, eccentricity $e$, argument of periastron $\omega$, orbital inclination $i$, longitude of the ascending node $\Omega$, and absolute planetary mass derived from fit (dependent on the LOS inclination). The argument of periastron is $\omega=\varpi-\Omega$, and for an edge-on or co-planar configuration $\Omega$ is undefined and thus $\omega=\varpi$. From the orbital period $P$ and assuming $m_{b, c} \sin i_{b . c} \ll M_{\star}$, the semi-major axes $a_{b, c}$ are calculated from the general form for the two-body problem:

$a_{b, c}=\left(\frac{G M_{\star} P_{b, c}^{2}}{4 \pi^{2}}\right)^{1 / 3}$.

Another input parameter is the Hill radius, which indicates the maximum distance from the body that constitutes a close encounter. A Hill radius approximation (Hamilton \& Burns 1992) is calculated from

$r_{b, c} \approx a_{b, c}\left(1-e_{b, c}\right)\left(\frac{m_{b, c}}{3 M_{\star}}\right)^{1 / 3}$.

All simulations were started from JD $=2451745.994$, the epoch when the first RV observation of $\eta$ Cet was taken, and then integrated for $10^{5}$ years. This timescale was chosen carefully to minimize CPU resources, while still allowing a detailed study of the system's evolution and stability. When a test system survived this period, we tested whether the system remains stable over a longer period of time by extending the integration time to $10^{8}$ years for the edge-on coplanar fits (Sect. 4.2), and to $2 \times 10^{6}$ years for inclined configurations (Sects. 4.3 and 4.4). On the other hand, the simulations were interrupted in case of collisions between the bodies involved in the test, or ejection of one of the planets. The typical time step we used for each dynamical integration was equal to eight days, while the output interval from the integrations was set to one year. We defined an ejection as one of the planet's semi-major axes exceeding 5 AU during the integration time.

In some cases none of the planets was ejected from the system and no planet-planet or planet-star collisions occurred, but because of close encounters, their eccentricities became very high and the semi-major axes showed single or multiple time planetary scattering to a different semi-major axis within the 5 AU limit. These systems showed an unpredictable behavior, and we classified them as chaotic, even though they may not satisfy the technical definition of chaos.

We defined a system to be stable if the planetary semi-major axes remained within $0.2 \mathrm{AU}$ from the semi-major axes values at the beginning of the simulation during the maximum integration time. This stability criterion provided us with a very fast and accurate estimate of the dynamical behavior of the system, and clearly distinguished the stable from the chaotic and the unstable configurations. In this paper we do not discuss the scattering (chaotic) configurations, but instead focus on the configurations that we qualify as stable. 


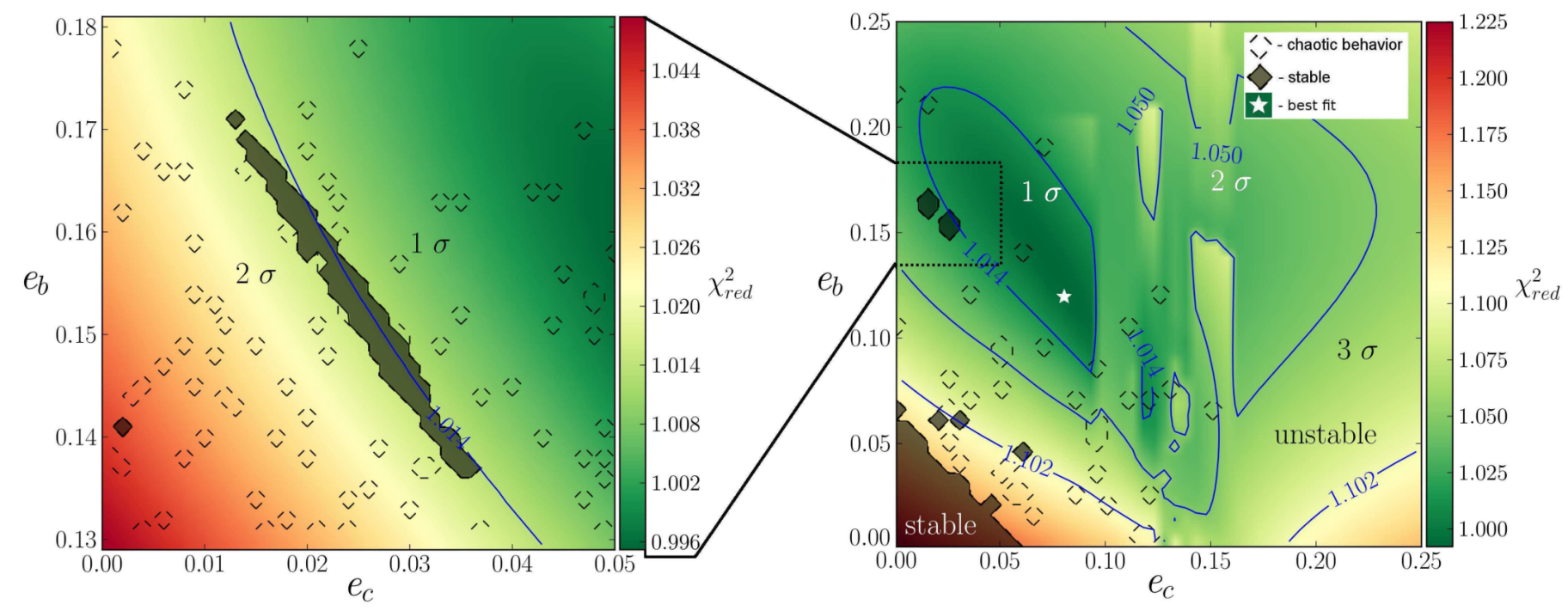

Fig. 5. Right: edge-on coplanar $\chi_{\text {red }}^{2}$ grid with jitter included. The eccentricities of both planets are varied in the range from 0.001 to 0.251 with steps of 0.005 , while the other orbital parameters and the zero-point offset were fitted until the $\chi_{\text {red }}^{2}$ minimum is achieved. The solid black contours indicate the stable fits, while the dashed contours indicate fits where the system survives the dynamical tests, but with chaotic scattering behavior. While the best dynamical fit is unstable (white star), we found two stability islands where long-term $\left(10^{8} \mathrm{yr}\right)$ stability is achieved. With a moderate $e_{b}$, at the $1 \sigma$ border (blue contours), a stable 2:1 MMR region exists, and at lower eccentricities a broad stability region can be seen at more than $3 \sigma$ from the best fit, without showing any signs of a low-order MMR. Left: higher resolution zoom of the stable 2:1 resonant region.

\subsection{Two-planet edge-on coplanar system}

The instability of the best fits motivated us to start a high-density $\chi_{\text {red }}^{2}$ grid search to understand the possible sets of orbital configurations for the $\eta$ Cet planetary system. To construct these grids we used only scaled $\chi_{\text {red }}^{2}$ fits with stellar jitter quadratically added, so that $\chi_{\text {red }}^{2}$ is close to unity. Later, we tested each individual set for stability, transforming these grids to effective stability maps.

In the edge-on coplanar two dimensional grid we varied the eccentricities of the planets from $e_{b, c}=0.001$ (to have access to $\varpi_{b, c}$ ) to 0.251 with steps of 0.005 (50 $\times 50$ dynamical fits), while the remaining orbital parameters in the model $\left(m_{b, c} \sin i_{b, c}\right.$, $P_{b, c}, M_{b, c}, \varpi_{b, c}$ and the RV offset) were fitted until the best possible solution to the data was achieved. The resulting $\chi_{\text {red }}^{2}$ grid is smoothed with bilinear interpolation between each grid pixel, and 1, 2 and $3 \sigma$ confidence levels (based on $\Delta \chi_{\text {red }}^{2}$ from the minimum) are shown (see Fig. 5). The grid itself shows that very reasonable $\chi_{\text {red }}^{2}$ fits can be found in a broad range of eccentricities, with a tendency toward lower $\chi_{\text {red }}^{2}$ values in higher and moderate eccentricities, and slightly poorer fits are found for the near-circular orbits. However, our dynamical test of the edge-on coplanar grid illustrated in Fig. 5 shows that the vast majority of these fits are unstable. The exceptions are a few isolated stable and chaotic cases, a large stable region at lower eccentricities, and a narrow stable island with moderate $e_{b}$, located about $1 \sigma$ away from the global minimum.

\subsubsection{Stable near-circular configuration}

It is not surprising that the planetary system has better chances to survive with near circular orbits. In these configurations, the bodies might interact gravitationally, but at any epoch they will be distant enough to not exhibit close encounters. By performing direct long-term $N$-body integrations, we conclude that the individual fits in the low-eccentricity region are stable for at least $10^{8}$ years, and none of them is involved in low-order MMR. Instead, the average period ratio of the stable fits in this region is between 1.8 for the very circular fits and 1.88 at the border of the stable region. This range of ratios is far away from the $2: 1 \mathrm{MMR}$, but could be close to a high-order MMR like 9:5, 11:6, 13:7, or even 15:8. However, we did not study these possible high-order resonances, and we assume that if not in $2: 1 \mathrm{MMR}$, then the planetary system is likely dominated by secular interactions.

The right column of Fig. 6 shows the dynamical evolution of the most circular fit from Fig. 5 with $\chi_{\text {red }}^{2}=14.1,\left(\chi_{\text {red }}^{2}=1.22\right)$, $m_{b} \sin i_{b}=2.4 M_{\mathrm{Jup}}, m_{c} \sin i_{c}=3.2 M_{\mathrm{Jup}}, a_{b}=1.28 \mathrm{AU}$, $a_{c}=1.94$ AU. We started simulations with $e_{b, c}=0.001$, and the gravitational interactions forced the eccentricities to oscillate very rapidly between 0.00 and 0.06 for $\eta$ Cet b, and between 0.00 to 0.03 for $\eta$ Cet c. The arguments of periastron $\omega_{b}$ and $\omega_{c}$ circulate between 0 and $360^{\circ}$, but the secular resonant angle $\Delta \omega_{b, c}=\omega_{c}-\omega_{b}$, while circulating, seems to spend more time around $180^{\circ}$ (anti-aligned). Within the stable near-circular region, the gravitational perturbations between the planets have lower amplitudes in the case of the most circular orbits than other stable fits with higher initial eccentricities (and smaller $\chi_{\text {red }}^{2}$ ).

The middle column of Fig. 6 illustrates the best $\chi_{\text {red }}^{2}$ fit within the low-eccentricity stable region with $\chi_{\text {red }}^{2}=13.03\left(\chi_{\text {red }}^{2}=1.13\right)$ and initial orbital parameters of $m_{b} \sin i_{b}=2.4 M_{\mathrm{Jup}}, m_{c} \sin i_{c}=$ $3.2 M_{\mathrm{Jup}}, a_{b}=1.28 \mathrm{AU}, a_{c}=1.93 \mathrm{AU}, e_{b}=0.06$, and $e_{c}=0.001$. The mean value of $\Delta \omega_{b, c}$ is again around $180^{\circ}$, while the amplitude is $\approx \pm 90^{\circ}$. Immediately after the start of the integrations $e_{c}$ has increased from close to 0.00 to 0.07 , and oscillates in this range during the dynamical test, while $e_{b}$ oscillates from 0.05 to 0.11 . In particular, the highest value for $e_{b}$ is very interesting because, as can be seen from Fig. 5, starting integrations with $0.08<e_{b}<0.11$ in the initial epoch yields unstable solutions. The numerical stability of the system appears to be strongly dependent on the initial conditions that are passed to the integrator. For different epochs the gravitational perturbations in the system would yield different orbital parameters than derived from the fit, and starting the integrations from an epoch forward or backward in time where $e_{b}$ or $e_{c}$ are larger than the $e_{b, c}=0.08$ limit might be perfectly stable. 
T. Trifonov et al.: Precise radial velocities of giant stars. VI.

2:1 MMR
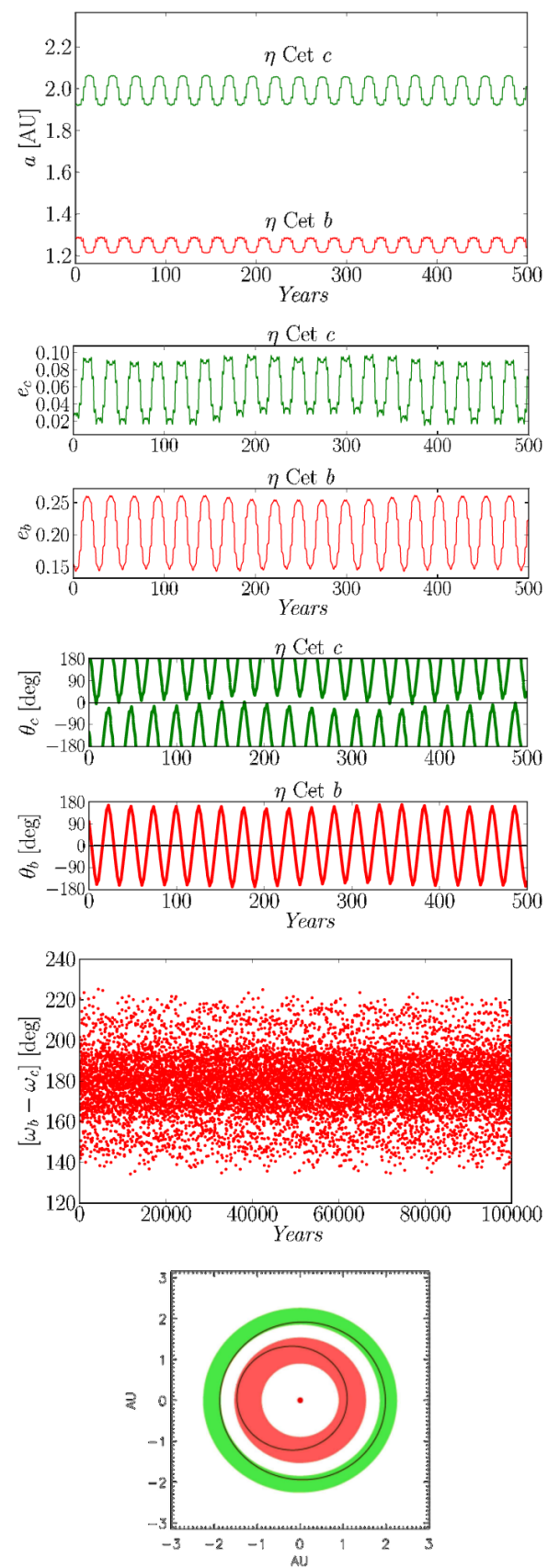

Near circular


Circular
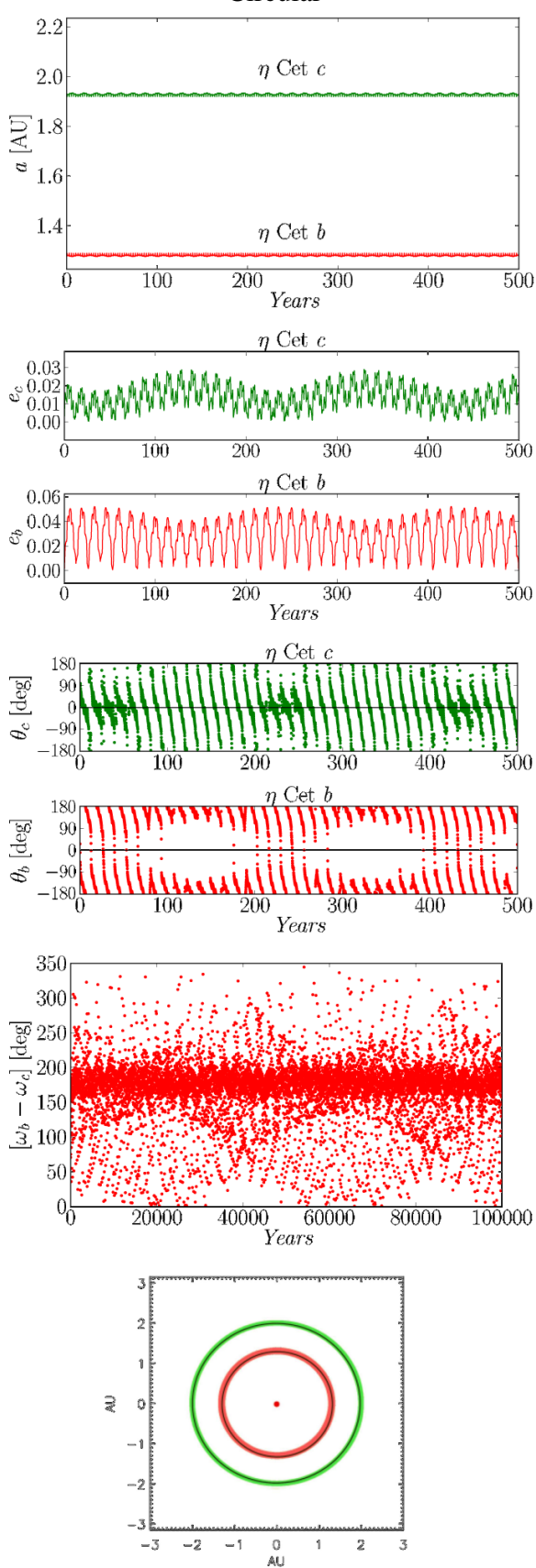

Fig. 6. Evolution of the orbital parameters for three different fits, stable for at least $10^{8}$ years. The best 2:1 MMR fit (left panels), the best stable fit from the low-eccentricity region (middle panels), and the fit with the most circular orbits (right panels). In the 2:1 MMR fit the gravitational perturbation between the planets is much larger than in the other two cases. It is easy to see that eccentricities for given epochs can be much larger than their values at the initial epoch of the integration. For convenience the evolution of the semi-major axes, eccentricities, the resonant angles (third row) and the $\Delta \omega_{b, c}$ are given for 500 and $10^{5}$ years, respectively. The bottom row gives the orbital precession region, the sum of orbits for each integration output.

We investigated the orbital evolution of a large number of fits in the low-eccentricity region and did not find any aligned system configuration. Instead, all systems studied with near circular configurations seem to settle in a secular resonance where $\Delta \omega_{b, c} \approx 180^{\circ}$ shortly after the start of the integrations, and exhibit a semichaotic behavior. This is expected as the system's secular resonance angle $\Delta \omega_{b, c}$ will circulate or librate, depending on the initial $\Delta \omega_{b, c}$ and eccentricity values at the beginning of the stability test (e.g., Laughlin et al. 2002; Lee \& Peale 2003). The fits in the near circular island always favor $\Delta \omega_{b, c} \approx 180^{\circ}$, and thus the system spends more time during the orbital evolution in this anti-aligned configuration.

\subsubsection{Narrow 2:1 MMR region}

The low-eccentricity island is located farther away from the best co-planar fit than the $3 \sigma$ confidence level, so we can neither consider it with great confidence nor reject the possibility 

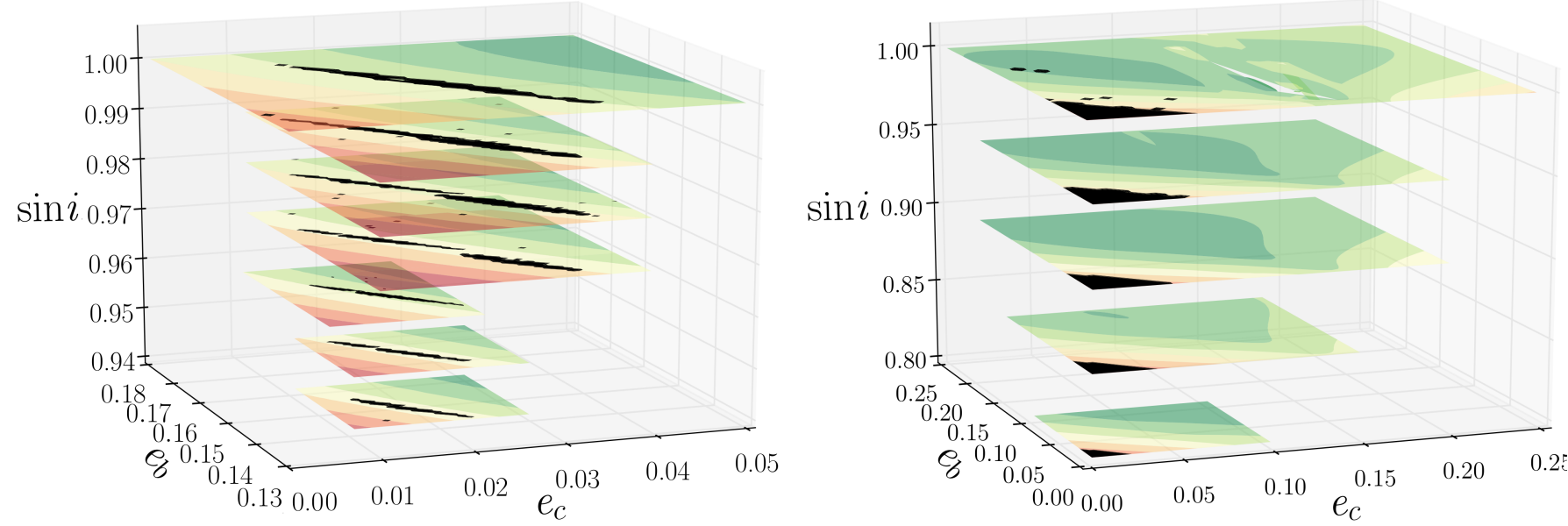

Fig. 7. Coplanar inclined grids illustrate the stability dependence on $m_{b, c} \sin i_{b, c}$. Color maps are the same as in Fig. 5, with the difference that for clarity, only the stable regions are shown (black). The top layer shows the grids from Fig. 5 , where $\sin i_{b, c}=1\left(i_{b, c}=90^{\circ}\right)$. Decreasing the inclination leads to smaller near-circular and 2:1 MMR stability regions. The resonant region shrinks and moves in the $\left(e_{b}, e_{c}\right)$ plane, and it completely vanishes when $\sin i \leq 0.93$. The stable island at low eccentricities vanishes for $\sin i \leq 0.75$, when even the most circular $\left(e_{b, c}=0.001\right)$ fit is unstable.

that the $\eta$ Cet system is perfectly stable in a near circular configuration. Thus, we focused our search for stable configurations on regions closer to the best fit. In the edge-on coplanar $\left(e_{b}, e_{c}\right)$ grid (Fig. 5), we have spotted a few fits at the $1 \sigma$ border that passed the preliminary $10^{5}$ years stability test. The additional long-term stability test proves that three out of four fits are stable for $10^{8}$ years.

To reveal such a set of stable orbital parameters, we created another high-density $\left(e_{b}, e_{c}\right)$ grid around these stable fits. We started with $0.131 \geq e_{b} \geq 0.181$ and $0.001 \geq e_{c} \geq 0.051$ with steps of $0.001(50 \times 50$ fits $)$. The significant increment of the resolution in this $\left(e_{b}, e_{c}\right)$ plane reveals a narrow stable island, where the vast majority of the fits have similar dynamical evolution and are stable for at least $10^{8}$ years (see left panel of Fig. 5).

The derived initial planetary periods for these fits are very close to those from the grid's best fit (Sect. 3.1) and initially do not suggest any low-order MMR. However, the mean planetary periods during the orbital evolution show that the system might be efficiently trapped in a 2:1 MMR. This result requires a close examination of the lowest order eccentricity-type resonant angles,

$\theta_{b}=\lambda_{b}-2 \lambda_{c}+\varpi_{b}, \quad \theta_{c}=\lambda_{b}-2 \lambda_{c}+\varpi_{c}$,

where $\lambda_{b, c}=M_{b, c}+\varpi_{b, c}$ is the mean longitude of the inner and outer planet, respectively. The resonant angles $\theta_{b}$ and $\theta_{c}$ librate around $\sim 0^{\circ}$ and $\sim 180^{\circ}$, respectively, for the whole island, leaving no doubt about the anti-aligned resonance nature of the system. $\theta_{b}$ and $\theta_{c}$ librate in the whole stable region with very large amplitudes of nearly $\pm 180^{\circ}$, so that the system appears to be very close to circulating (close to the separatrix), but in an antialigned planetary configuration, where the secular resonance angle $\Delta \omega=\theta_{b}-\theta_{c}=\omega_{b}-\omega_{c}$ librates around $180^{\circ}$. A similar behavior was observed during the first 17000 years of orbital evolution of the best edge-on coplanar fit from Sect. 3.1. These results suggest that systems in the $\left(e_{b}, e_{c}\right)$ region around the best edge-on fit are close to 2:1 MMR, but also appear to be very fragile, and only certain orbital parameter combinations may lead to stability.

Figure 6 (left column) illustrates the orbital evolution of the system with the best stable fit from the resonant island; the initial orbital parameters can be found in Table 3. The eccentricities rapidly change with the same phase, reaching moderate levels of $e_{b}=0.15 \ldots 0.25$ and $e_{c}=0.0 \ldots 0.08$, while the planetary semi-major axes oscillate in opposite phase, between $a_{b}=1.21 \ldots 1.29 \mathrm{AU}$ and $a_{c}=1.92 \ldots 2.06 \mathrm{AU}$. During the dynamical test over $10^{8}$ years $\Delta \omega_{b, c}$ librates around $180^{\circ}$ with an amplitude of $\approx \pm 15^{\circ}$, while the $\omega_{b}$ and $\omega_{c}$ are circulating in an anti-aligned configuration.

\subsection{Coplanar inclined system}

To create coplanar inclined grids we used the same technique as in the edge-on coplanar grids, with additional constraints $i_{b}=i_{c}$ and $i_{b, c} \neq 90^{\circ}$. We kept $\Omega_{b, c}=0^{\circ}$ as described in Sect. 3.2, so that we get an explicitly coplanar system. A set of grids concentrated only on the 2:1 MMR stable island are shown in Fig. 7 (left panel), and larger grids for the low-eccentricity region are shown in the right panel of Fig. 7. For reference, we placed the two grids from Fig. 5 with $\sin i=1$ at the top of Fig. 7. This coplanar inclined test shows how the stable islands behave if we increase the planetary masses via the LOS inclination. We limited the grids with $\sin i \neq 1$ in Fig. 7 to a smaller region in the $\left(e_{b}, e_{c}\right)$ plane than the grids from Fig. 5 to use CPU time efficiently, focusing on the potentially stable $\left(e_{b}, e_{c}\right)$ space and avoiding highly unstable fit regions.

The smaller grid area is compensated for by higher resolution, however, as those grids have between 3600 and 10000 fits. For simplicity we do not show the chaotic configurations, but illustrate only the stable fits that survived the maximum evolution span of $10^{5}$ years (dark areas) for this test in Fig. 7. We studied the near circular stable region by decreasing $\sin i$ with a step size of 0.05 in the range from 1 to 0.80 . The five stability maps of Fig. 7 (right panel) clearly show the tendency that the near circular stable region becomes smaller when the planetary mass increases with decreasing $\sin i$. The stable island near circular orbits preserves its stability down to $\sin i \approx 0.75(i \sim 49 \mathrm{deg})$, when even the most circular fit $\left(e_{b, c}=0.001\right)$ becomes unstable.

The 2:1 MMR region strongly evolves and also decreases in size while decreasing $\sin i$. When $\sin i \approx 0.94\left(i \approx 70^{\circ}\right)$, the 2:1 MMR region is smallest and completely vanishes when 




Fig. 8. Mutually inclined grids where $\Omega_{b, c}=0$, and the mutual inclination comes only from $\Delta i_{b, c}=i_{b}-i_{c}$. The low-eccentricity stable island increases its size by assuming higher mutual inclinations, eventually creating an overlap with the $1 \sigma$ confidence level from the grid's best fit (dark green contours).

$\sin i \leq 0.93$. We find that the largest stable area is reached for $\sin i=1$, and thus, there is a high probability that the $\eta$ Cet system is observed nearly edge-on and involved in an anti-aligned 2:1 MMR. The repeated tests with $S y M B A$ for $2 \times 10^{6}$ years are consistent with the Mercury results, although the stability regions were somewhat smaller. This is due to the longer simulations, which eliminate the long-term unstable fits. Integrating for $10^{8}$ years may leave only the true stable central regions, however, such a long-term dynamical test over the grids requires much longer CPU time than we had for this study.

\subsection{Mutually inclined system}

Constraining the mutual inclination from the RV data alone is very challenging, even for well known and extensively studied extrasolar planetary systems, and requires a large set of highly precise RV and excellent astrometric data (see Bean \& Seifahrt 2009). For $\eta$ Cet, the number of radial velocity measurements is relatively small, so that we cannot derive any constraints on the mutual inclination from the RV. We tried to derive additional constraints on the inclinations and/or the ascending nodes of the system from the HIPPARCos Intermediate Astrometric Data, as was done in Reffert \& Quirrenbach (2011) for other systems. We found that all but the lowest inclinations (down to about $5^{\circ}$ ) are consistent with the HIPPARCos data, so no further meaningful constraints could be derived.

We have shown in Sects. 4.2 and 4.3 that we most likely observe the $\eta$ Cet planetary system nearly edge-on, because we found a maximum of stable fits at $\sin i=1$, in line with the HIPPARCos constraint. Assuming lower inclinations, the size of the stability region in the $\left(e_{b}, e_{c}\right)$ plane decreases. Because we constrained the system inclination only by stability criteria, it would be interesting to see whether the stability will sufficiently increase if we allow a mutual inclination between the orbits to occur in our fits, or whether the system will become more unstable than for the coplanar fits. Moreover, as we have shown in Sect. 3.2, by adding three additional fitting parameters we obtain significantly better fits (although very unstable), and it is important to determine whether we can find any stable solutions or even stable islands for highly inclined non-coplanar configurations.

To study the dynamics of the $\eta$ Cet system for mutually inclined orbits we investigated the stability with the following three different strategies:

1) We fixed the $\Delta i_{b, c}$ to be constant and assumed $\Omega_{b, c}=0^{\circ}$, so that the mutual inclination depends only on $i_{b, c}$. We defined $\Delta i_{b, c}=i_{b}-i_{c}$, where for the $2: 1 \mathrm{MMR}$ region the $i_{b}=89.5^{\circ}$, $89^{\circ}, 88.5^{\circ}$ and $i_{c}=90.5^{\circ}, 91^{\circ}, 91.5^{\circ}$. We found that the size of the 2:1 MMR stable region decreases very fast with mutual inclination, and after $\Delta i_{b, c}>2^{\circ}$ the $2: 1 \mathrm{MMR}$ island completely vanishes. For the global $\left(e_{b}, e_{c}\right)$ grid we defined $i_{b}=85^{\circ}, 80^{\circ}, 75^{\circ}$ and $i_{c}=95^{\circ}, 100^{\circ}, 105^{\circ}$. The results from this test are illustrated in Fig. 8. From the grid we found that the low-eccentricity stable region shows a trend of expanding its size with the mutual inclination for $\Delta i_{b, c}=10^{\circ}$ and $20^{\circ}$. When $\Delta i_{b, c}=30^{\circ}$ the stable area expands and we find many stable fits for moderate $e_{c}$ within the $1 \sigma$ confidence region from the $\Delta i_{b, c}=30^{\circ}$ grid. This test shows that for a high mutual inclination there is a high probability for the system to have near circular orbits, or moderate $e_{c}$.

2) We again relied on the $\left(e_{b}, e_{c}\right)$ grids, where additional free parameters in the fits are $i_{b, c}$ and $\Omega_{b, c}$. However, in the fitting we restricted the minimum inclination to be $i_{b, c}=90^{\circ}$, and the $\sin i_{\max }$ factor comes only from inclination angles above this limit. In this test we only studied the global $\left(e_{b}, e_{c}\right)$ plane, without examining the 2:1 MMR region separately. Initially, for the first grid we set $\sin i_{\max }=0.707$ $\left(i_{\max }=135^{\circ}\right)$, while $\Omega_{b, c}$ was unconstrained and was let to vary across the full range from 0 to $2 \pi$. Later we constructed grids by decreasing the maximum allowed inclination to $\sin i_{\max }=0.819,0.906,0.966$ and at last 0.996 $\left(i_{\max }=125^{\circ}, 115^{\circ}, 105^{\circ}\right.$, and $95^{\circ}$, respectively $)$, thereby decreasing the upper limit on the planetary masses and mutual inclination angle $\Delta i_{b, c}$. In all grids we allowed $i_{b} \neq i_{c}$, and $\Omega_{b} \neq \Omega_{c}$ as we discussed in Sect. 3.2. We find that when increasing $i_{\text {max }}$, the grid's $\chi_{\text {red }}^{2}$ values improved, and the planetary $i_{b, c}$ are usually close to $i_{\max }$. The obtained $\Delta \Omega_{b, c}$ is also very low, favoring low mutual inclinations in this test. When $i_{\max }=95^{\circ}$ the average mutual inclination over the grid is $0.22^{\circ}$, making the grid nearly coplanar. In this case the planetary masses are only $\sim 0.5 \%$ higher than their minimum, which has a negligible effect on the stability. However, the very small orbital misalignment in those systems seems to have a positive influence on the system stability, and we find slightly more stable fits than for the edge-on coplanar case discussed in Sect. 4.2 (see Fig. 9).

At larger $i_{\max }$ the $\chi_{\text {red }}^{2}$ distribution in general has lower values, but the size of the stability region is decreasing. This is probably due to the increased planetary masses and the stronger gravitational perturbations in the dynamical simulations. There is not even one stable solution at $i_{\max }=135^{\circ}$, but we have to note that there are many chaotic fits that survived the $10^{5} \mathrm{yr}$ test, which are not shown in Fig. 9.

3) Because of the resulting low mutual inclination in the second test, we decided to decouple the planetary orbits and to test for higher mutual inclinations by allowing $\Delta \Omega_{b, c} \neq 0$, in contrast to the first test where $\Delta \Omega_{b, c}=0$. We constructed a grid of best fits for fixed $i_{b, c}\left(i_{b}, i_{c}\right.$ grid $)$. The planetary inclination $i_{b, c}$ was increased from $90^{\circ}$ to $140^{\circ}$ with steps of $1^{\circ}$, while rest of the orbital parameters were free in the fitting. This test attempts to check for stability for almost all the possible mutual inclinations in the system. Figure 10 illustrates the 


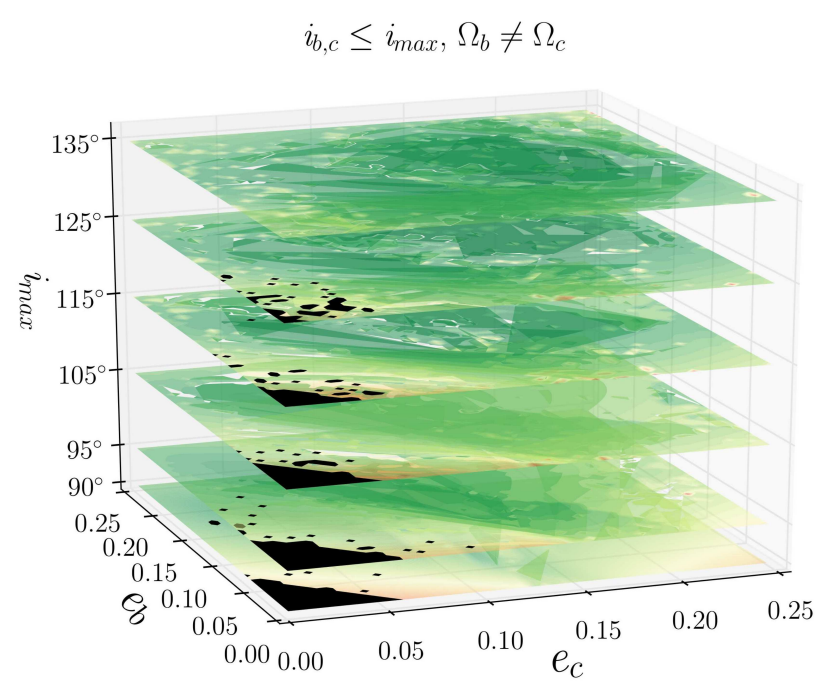

Fig. 9. Grids with mutual inclination dependent on $\Delta \Omega_{b, c}$ and $i_{b, c} \leq i_{\max }$. During the fitting the mutual inclinations from the grids are very low, on the order of $\sim 3^{\circ}$. The stability area has a maximum at $i_{\max }=95^{\circ}$, slightly higher than the coplanar grid at $90^{\circ}$. The stability decreases at larger $i_{\max }$ due to the larger planetary masses and the stronger perturbations. There is no stable solution at $i_{\max }=135^{\circ}$.

stability output in the $\left(i_{b}, i_{c}\right)$ plane. The $\chi_{\text {red }}^{2}$ solutions in this grid have lower values when $i_{b} \approx i_{c}$ (close to the coplanar configuration), and have a clear trend of better fits when the LOS inclination is high. However, we could not find any stable configuration near the coplanar diagonal of best fits from $i_{b, c}=90^{\circ}$ to $140^{\circ}$. There are many chaotic survivals and few stable islands at higher mutual inclinations, more than $3 \sigma$ away from the grid minimum.

We are aware of the fact that, when including the $i_{b, c}$ and $\Omega_{b, c}$ in the fitting model, the parameter space becomes extremely large, and perhaps many more smaller and possibly stable minima might exist, but we have not been able to identify any in this study.

\subsection{Impact of stellar mass on the stability analysis}

Finally, we have tested how changing the assumed stellar mass influences the stability in $\left(e_{b}, e_{c}\right)$ space. We generated coplanar edge-on $\chi_{\text {red }}^{2}$ grids by assuming different stellar masses, using the values in Table 1 . We took the same $\left(e_{b}, e_{c}\right)$ grid area and resolution as discussed in Sect. 4.2 (Fig. 5) and started our grid search with $1 M_{\odot}$ and $1.4 M_{\odot}$, which are the lower and upper stellar mass limits for $\eta$ Cet proposed by Berio et al. (2011). Next we assumed $1.3 M_{\odot}$ from Luck \& Challener (1995), then $1.84 M_{\odot}$, which is the upper limit from Reffert et al. (2014), and we already have the $1.7 M_{\odot}$ grid from Sect. 4.2. The longest integration time applied to the stability test for these grids was $10^{5}$ years.

Stability results are shown in Fig. 11, which clearly shows a trend of higher stability with larger stellar mass. Our starting mass of $1 M_{\odot}$ leads almost to the disappearance of the low-eccentricity stable island, and only a few stable solutions can be seen for very circular orbits. Increasing the stellar mass to $1.3 M_{\odot}, 1.4 M_{\odot}$ and $1.7 M_{\odot}$ reveals larger stable areas at low eccentricities. Finally, in the $1.84 M_{\odot}$ grid we see the largest low-eccentricity stable island in our test.

Similarly, the 2:1 MMR region evolves and decreases in size with decreasing $\eta$ Cet mass. No resonant island is seen at $1 M_{\odot}$.

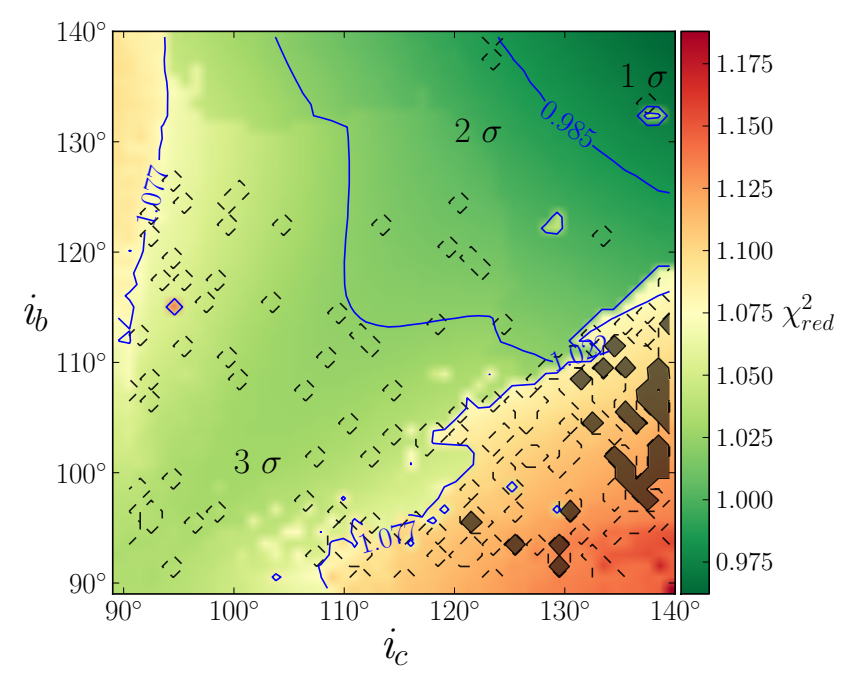

Fig. 10. Except for the inclinations, the remaining orbital parameters are free to vary for the $\left(i_{b}, i_{c}\right)$ grid $(50 \times 50$ fits $)$. The $\chi_{\text {red }}^{2}$ solutions suggest a higher LOS inclination and close to coplanar configurations. There are no obvious stable solutions near the coplanar diagonal of best fits from $i_{b, c}=90^{\circ}$ to $140^{\circ}$ ). Instead, there are many chaotic survivals and few stable islands at higher mutual inclinations, more than $3 \sigma$ away from the grid minimum.

However, the 2:1 MMR region has a larger area at $1.7 M_{\odot}$ than in the $1.84 M_{\odot}$ grid.

This effect comes from the fact that starting the fitting process with lower stellar mass will also scale down the whole planetary system. The derived orbital angles and the periods from the dynamical fitting will remain almost the same in the $\left(e_{b}, e_{c}\right)$ grids when fitting from $1 M_{\odot}$ to $1.84 M_{\odot}$. However, as can be seen from Eqs. (1), (3) and (4), the planetary masses, semi-major axes $a_{b, c}$, and the Hill radii $r_{b, c}$ are dependent on the stellar mass. By scaling down the planetary masses, we would expect the gravitational interactions between the planets to be less destructive, and we would thus expect more stable fits when adopting a lower mass for the primary. This is exactly the opposite of what can be seen in Fig. 11. The dynamical simulations are much more sensitive to $\Delta a_{b, c}=a_{c}-a_{b}$ than to the planetary mass ratio. From Eqs. (4) and (5) it can be seen that $r_{b, c}$ will slightly decrease when assuming a lower stellar mass, although not as fast as $a_{b, c}$ will decrease. $\Delta a_{b, c}$ for the $1 M_{\odot}$ grid is on average $\approx 0.54 \mathrm{AU}$, and for the $1.84 M_{\odot}$ grid $\approx 0.65 \mathrm{AU}$. The more similar planetary semi-major axes in lower stellar mass systems lead to a higher number of close encounters during the orbital evolution, and thus, to a higher ejection rate, especially for fits with higher $e_{b, c}$. On the other hand, the $\Delta a_{b, c}$ for the grid with maximum mass for $\eta$ Cet $\left(1.84 M_{\odot}\right)$ is enough to keep the two planets well separated, and the stability region in lower eccentricities increases.

\section{Discussion}

\subsection{Planetary hypothesis}

In principle, the possible reasons for observed RV variability in $\mathrm{K}$ giants are rotational modulation of star spots, long-period nonradial pulsations, or the presence of planets.

Star spots can most likely be excluded as a viable explanation for the observed RV variability of $\eta$ Cet, at least for one of the two observed periods. If star spots were to cause the RV to vary, one of the two periods that we observe would 
T. Trifonov et al.: Precise radial velocities of giant stars. VI.



Fig. 11. Stability maps with different initial masses for $\eta$ Cet. A clear stability trend can be seen in the $\left(e_{b}, e_{c}\right)$ grids in the sense that by increasing the stellar mass up to the maximum of $1.84 M_{\odot}$, the size of the stable region increases as well.

have to match the rotational period (still leaving the second period unexplained). However, the longest rotation period of $\eta$ Cet compatible with its radius of $R=14.3 \pm 0.2 R_{\odot}$ and its projected rotational velocity $v \sin i=3.8 \pm 0.6 \mathrm{~km} \mathrm{~s}^{-1}$ (Hekker \& Meléndez 2007) is $190_{-26}^{+36}$ days. This is much shorter than either the 405-day or the 750-day period. Moreover, one would expect a larger photometric variation than the 3 mmag observed by HIPPARCOS for a star spot to generate an RV amplitude on the order of $50 \mathrm{~m} \mathrm{~s}^{-1}$ (Hatzes \& Cochran 2000) $)^{3}$. On the other hand, macro-turbulent surface structures on $\mathrm{K}$ giants are currently poorly understood. Large and stable convection cells could act as velocity spots, yielding an RV variability without significant photometric variability (e.g., Hatzes \& Cochran 2000). Although unlikely, we cannot fully exclude that the shorter 405-day period is due to rotational modulation of surface features, while the longer 750-day period is most certainly due to a planet.

Ruling out nonradial pulsations is harder than ruling out star spots, but also possible. First, we see evidence of eccentricity in the shape of the radial velocity curves, which is an indication for a Keplerian orbit. Second, the signal has been consistent over 12 years; this is not necessarily expected for pulsations. Third, on top of the optical RV data we derived the RV from the infrared wavelength regime (CRIRES). Although the IR RV error is much larger than that from the optical data, the CRIRES data are clearly consistent with the Lick data. The best dynamical fit derived from the visible data and applied only to the near-IR data has $\chi_{\text {red }}^{2}=0.502$ and $\mathrm{rms}=26.7 \mathrm{~m} \mathrm{~s}^{-1}$, while a constant model assuming no planets has $\chi_{\text {red }}^{2}=2.806$ and $\mathrm{rms}=63.2 \mathrm{~m} \mathrm{~s}^{-1}$. Therefore, the two-planet fit is more likely. Just based on the CRIRES data, we can rule out infrared amplitudes smaller than $30 \mathrm{~m} \mathrm{~s}^{-1}$ or larger than $65 \mathrm{~m} \mathrm{~s}^{-1}$ with $68.3 \%$ confidence. If the RV variability in the optical were to be caused by pulsations, one might expected to find a different amplitude in the IR, which is not the case. Fourth, there are no indications for these long-period nonradial pulsations in $\mathrm{K}$ giants yet, although a variety of very short pulsation modes have been found

\footnotetext{
The HIPPARCos observations were taken earlier (until 1993) than our $\mathrm{RV}$ data (since 2000), but assuming that $\eta$ Cet was quiet for four years, and active with a very constant RV amplitude for more than ten years just seven years later appears rather contrived.
}

with Kepler (Bedding et al. 2011). Taken together, there is no supporting evidence for the presence of pulsations in $\eta$ Cet.

The strongest evidence supporting a multiple planetary system comes from the dynamical modeling of the RV data. Taking the gravitational interactions between the two planets into account leads to a considerably better fit than the two Keplerian model (or two sinusoids). In other words, we were able to detect the interactions between the two planets in our data, which strongly supports the existence of the two planets.

Thus, though we cannot completely rule out alternative explanations, a two-planet system is the most plausible interpretation for the observed RV variations of $\eta$ Cet.

\subsection{Giant star planetary population}

With the detection of the first extrasolar planet around the giant star $\iota$ Dra b (the first planet announced from our Lick sample - Frink et al. 2002) the search for planets around evolved intermediate mass stars has increased very rapidly. Several extrasolar planet search groups are working in this field to provide important statistics for planet occurrence rates as a function of stellar mass, evolutionary status, and metallicity (Frink et al. 2001; Sato et al. 2003; Hatzes et al. 2006; Niedzielski et al. 2007; Döllinger et al. 2009; Mitchell et al. 2013, etc.). Up to date, there are 56 known planets around 53 giants stars in the literature, and all of them are in wide orbits.

Except for the $\eta$ Cet discovery, there are only three candidate multiple planetary systems known to orbit giant stars, and all of them show evidence of two massive substellar companions. Niedzielski et al. (2009a,b) reported planetary systems around the K giants HD 102272 and BD +20 2457. While the published radial velocity measurements of HD 102272 are best modeled with a double Keplerian, the sparse data sampling is insufficient to derive an acurate orbit for the second planet. Initial stability tests based on the published orbital parameters show a very fast collision between the planets. This appears to be due to the presumably very eccentric orbit of the outer planet, which leads to close encounters, making the system unstable. The case of $\mathrm{BD}+202457$ is even more dramatic. The bestfit solution suggests a brown dwarf and a very massive planet just at the brown dwarf mass border, with minimum masses of $m_{1} \sin i_{1} \approx 21.4 M_{\text {Jup }}$ and $m_{2} \sin i_{2} \approx 12.5 M_{\text {Jup }}$, respectively ( 1 is the inner and 2 the outer planet). This system has very similar orbital periods of $P_{1} \approx 380$ days and $P_{2} \approx 622$ days for such a massive pair, and the gravitational interactions are extremely destructive. We have tested several orbital parameter sets within the derived parameter errors and, without conducting any comprehensive stability analysis, so far we were unable to find any stable configuration for BD +20 2457. Recently, Horner et al. (2014) investigated the stability of BD +20 2457 in more detail and did not find any stable solutions either. However, we have to keep in mind that the formal best fit for $\eta$ Cet was also unstable, and only after an extensive stability analysis did we find longterm stable regions. In this context we do not exclude the possibility that HD 102272 and BD +20 2457 indeed harbor multiple planetary systems, but better data sampling is required to better constrain the planetary orbits. Moreover, additional efforts must be undertaken to prove the stability for these two systems, perhaps including highly mutually inclined configurations, or even better constraints on the stellar masses.

Of particular interest is also the system around the $\mathrm{K}$ giant $v$ Oph (Quirrenbach et al. 2011; Sato et al. 2012), which is consistent with two brown dwarfs with masses $m_{1} \sin i_{1} \approx$ $22.3 M_{\text {Jup }}, m_{2} \sin i_{2} \approx 24.5 M_{\text {Jup }}$. The two brown dwarfs exhibit 
a clear 6:1 MMR, with periods of $P_{1} \approx 530$ days and $P_{2} \approx$ 3190 days.

Although $v$ Oph and potentially BD +20 2457 are not planetary systems, they may present important evidence for brown dwarf formation in a circumstellar disk. Such objects may form because in general the more massive stars should have more massive disks from which protoplanetary objects can gain enough mass to become brown dwarfs. It might be possible for the 6:1 resonance configuration of $v$ Oph to have formed via migration capture in a protoplanetary disk around a young intermediate-mass progenitor, and the brown dwarf occurrence may be rather high (Quirrenbach et al. 2011).

Therefore, if we exclude $v$ Oph, which is clearly a brown dwarf system, and the HD 102272 and BD +20 2457 systems, which suffer from poor data sampling and stability problems, $\eta$ Cet is currently the only $\mathrm{K}$ giant star that shows strong evidence for harboring a stable multiplanetary system.

\subsection{Unique orbital configuration of $\eta$ Cet}

The stable solutions from the 2:1 MMR region in the edge-on coplanar and inclined tests raise some important questions about the possible formation and evolution of the $\eta$ Cet planetary system. The $\theta_{b} \approx 0^{\circ}$ and $\theta_{c} \approx 180^{\circ} 2: 1$ MMR configuration is similar to that of the $2: 1$ resonance between the Jovian satellites Io and Europa, but the Io-Europa configuration is not supposed to exist for relatively large eccentricities (see Beaugé et al. 2003; Lee 2004). The average $e_{b}(\sim 0.2)$ and $e_{c}(\sim 0.05)$ for $\eta$ Cet are much larger than the $e_{b}, e_{c}$ boundary where the $\theta_{b} \approx 0^{\circ}$ and $\theta_{c} \approx 180^{\circ}$ configuration exists. An aligned configuration with both $\theta_{b}$ and $\theta_{c}$ librating about $0^{\circ}$ is expected for a mass ratio $m_{b} / m_{c} \approx 0.77$ and the eccentricities in the 2:1 MMR region.

One possible stabilizing mechanism for the $\eta$ Cet system might be the large libration amplitudes of both $\theta_{b}$ and $\theta_{c}$, which are almost circulating. We made some preliminary attempts and failed to find a small libration amplitude counterpart. More work is needed to understand the stability of this $2: 1$ MMR configuration, as well as its origin, if $\eta$ Cet is indeed in this configuration.

We cannot fully exclude that the true system configuration of $\eta$ Cet corresponds to some of the single isolated stable fits that we see in the stability maps, and neither can we exclude one of the numerous fits that are stable for $10^{8}$ years, which show a scattering chaotic behavior. A nonresonant system in near circular orbits is also possible.

\section{Summary}

We have reported the discovery of a planetary system around the $\mathrm{K}$ giant star $\eta$ Cet. This discovery is the result of a long-term survey, which aims to discover planetary companions around 373 intermediate-mass $\mathrm{G}$ and $\mathrm{K}$ giant stars, and which started back in 1999 at Lick Observatory. We presented 118 highprecision optical radial velocities based on the observations with the Hamilton spectrograph at Lick Observatory and nine nearinfrared data points from the ESO CRIRES spectrograph; these data cover more than a decade.

We have fitted a dynamical model to the optical data, which ensured that any possible gravitational interactions between the planets are taken into account in the fitting process. We showed that the dynamical model represents a significant $\chi_{\text {red }}^{2}$ improvement over the double-Keplerian fit.

In an attempt to characterize the most likely planetary configuration, we performed an extensive stability analysis of the $\eta$ Cet system. We made a wide variety of high-resolution coplanar and inclined dynamical $\chi_{\text {red }}^{2}$ grids, which we used as an input for our numerical analysis. Thus, we transformed these grids into detailed stability maps. In total, we carried out more than 200000 dynamical integrations with typical time spans of $10^{5}$ and $2 \times 10^{6}$ years, and we extended the test to $10^{8}$ years to study the edge-on coplanar case.

For the edge-on coplanar grid we used a set of best fits for fixed $e_{b}$ and $e_{c}$. We found that the $\eta$ Cet system can be stable for at least $10^{8}$ years, locked in a 2:1 MMR in a region with moderate $e_{b}$, which lies about $1 \sigma$ away from the best co-planar fit. A much larger nonresonant stable region exists with nearly circular orbits, although it is located more than $3 \sigma$ away from the best fit and is thus less likely. In the 2:1 MMR region all fits are in an anti-aligned planetary configuration and very close to the separatrix. The low-order eccentricity-type resonant angles $\theta_{b}$ and $\theta_{c}$ librate around $0^{\circ}$ and $180^{\circ}$, respectively, but with very large amplitudes of $\approx \pm 180^{\circ}$. A similar near-separatrix behavior can be seen in the stable fits with near circular orbits, where the secular resonance angle $\Delta \omega_{b, c}$ circulates, but during most of the simulation the planetary configuration is anti-aligned $\left(\Delta \omega_{b, c} \approx 180^{\circ}\right)$.

We provided a detailed set of $\left(e_{b}, e_{c}\right)$ coplanar inclined stability maps, showing that the $\eta$ Cet system is very likely observed in a near edge-on configuration $\left(i_{b, c} \approx 90^{\circ}\right)$. The size of the stable region is largest when the system is assumed to have $i_{b, c}=90^{\circ}$, and when we increased the planetary mass via $\sin i_{b, c}$, the size of the two stability regions in the $\left(e_{b}, e_{c}\right)$ plane decreased. The 2:1 MMR stable island totally disappeared when $i_{b, c} \approx 70^{\circ}$, while the near circular stable island survived until the LOS inclination becomes $i_{b, c} \sim 49^{\circ}$. Below the last inclination limit, all the fits in the $\left(e_{b}, e_{c}\right)$ plane became unstable.

We also presented results from a grid based on mutually inclined configurations, although we pointed out that they need not be exhaustive. This is because of the large amount of computational time needed when the parameter space is expanded. Another way of constraining the mutual inclination would be a very extensive and precise radial velocity and astrometric data set, which is so far not available for $\eta$ Cet.

The most important conclusion from the inclined dynamical test is that the planets cannot be more massive than a factor of $\sim 1.4$ heavier than their suggested minimum masses. Higher inclinations, and thus larger planetary masses, lead to instability in all cases. This excludes the possibility of two brown dwarfs purely based on stability considerations, and strongly favors a planetary system.

We also tested how the uncertainty of the stellar mass will affect the dynamical stability of the system. Decreasing the stellar mass leads to a smaller size of the stable region in the $\left(e_{b}, e_{c}\right)$ grids, and thus we conclude that the stellar mass value from Reffert et al. (2014) is indeed a very reasonable estimate.

The $\eta$ Cet system is only the fourth candidate multiple substellar system around a $\mathrm{G}$ or $\mathrm{K}$ giant star and presents an important milestone for understanding planetary formation and evolution as a function of stellar mass, metallicity, and age.

Acknowledgements. Part of this work was supported by the International Max Planck Research School for Astronomy and Cosmic Physics at the University of Heidelberg, IMPRS-HD, Germany. M.H.L. was supported in part by the Hong Kong RGC grant HKU 7024/13P. We would like to thank the staff at Lick Observatory for their support over the years of this project. We kindly thank the CAT observers that assisted with this project, including Saskia Hekker, Simon Albrecht, David Bauer, Christoph Bergmann, Stanley Browne, Kelsey Clubb, Dennis Kügler, Christian Schwab, Julian Stürmer, Kirsten Vincke, and Dominika Wylezalek. We thank Mathias Zechmeister and Ansgar Reiners for their help with the acquisition and reduction of the CRIRES data. We thank 
T. Trifonov et al.: Precise radial velocities of giant stars. VI.

Stefano Meschiari for the very helpful discussion regarding the capabilities of the Console package. We would also like to thank our referee, Artie Hatzes, for his constructive comments that helped to improve this paper.

\section{References}

Baranne, A., Queloz, D., Mayor, M., et al. 1996, A\&AS, 119, 373

Barban, C., De Ridder, J., Mazumdar, A., et al. 2004, in SOHO 14 Helio- and

Asteroseismology: Towards a Golden Future, ed. D. Danesy, ESA SP, 559, 113

Bean, J. L., \& Seifahrt, A. 2009, A\&A, 496, 249

Bean, J. L., Seifahrt, A., Hartman, H., et al. 2010, ApJ, 711, L19

Beaugé, C., Ferraz-Mello, S., \& Michtchenko, T. A. 2003, ApJ, 593, 1124

Bedding, T. R., Mosser, B., Huber, D., et al. 2011, Nature, 471, 608

Berio, P., Merle, T., Thévenin, F., et al. 2011, A\&A, 535, A59

Butler, R. P., Marcy, G. W., Williams, E., et al. 1996, PASP, 108, 500

Butler, R. P., Marcy, G. W., Williams, E., Hauser, H., \& Shirts, P. 1997, ApJ, 474, L115

Butler, R. P., Marcy, G. W., Fischer, D. A., et al. 1999, ApJ, 526, 916

Chambers, J. E. 1999, MNRAS, 304, 793

Correia, A. C. M., Udry, S., Mayor, M., et al. 2009, A\&A, 496, 521

De Ridder, J., Barban, C., Carrier, F., et al. 2006, A\&A, 448, 689

Desort, M., Lagrange, A.-M., Galland, F., et al. 2008, A\&A, 491, 883

Döllinger, M. P., Hatzes, A. P., Pasquini, L., et al. 2009, A\&A, 499, 935

Duncan, M. J., Levison, H. F., \& Lee, M. H. 1998, AJ, 116, 2067

Figueira, P., Pepe, F., Melo, C. H. F., et al. 2010, A\&A, 511, A55

Fischer, D. A., Marcy, G. W., Butler, R. P., Laughlin, G., \& Vogt, S. S. 2002, ApJ, 564, 1028

Fischer, D. A., Marcy, G. W., Butler, R. P., et al. 2003, ApJ, 586, 1394

Fischer, D. A., Marcy, G. W., Butler, R. P., et al. 2008, ApJ, 675, 790

Ford, E. B. 2005, AJ, 129, 1706

Frandsen, S., Carrier, F., Aerts, C., et al. 2002, A\&A, 394, L5

Frink, S., Quirrenbach, A., Fischer, D., Röser, S., \& Schilbach, E. 2001, PASP, 113,173

Frink, S., Mitchell, D. S., Quirrenbach, A., et al. 2002, ApJ, 576, 478

Gaudi, B. S., Bennett, D. P., Udalski, A., et al. 2008, Science, 319, 927

Girardi, L., Bressan, A., Bertelli, G., \& Chiosi, C. 2000, A\&AS, 141, 371

Gray, R. O., Corbally, C. J., Garrison, R. F., et al. 2006, AJ, 132, 161

Hamilton, D. P., \& Burns, J. A. 1992, Icarus, 96, 43

Hatzes, A. P., \& Cochran, W. D. 2000, AJ, 120, 979

Hatzes, A. P., Cochran, W. D., Endl, M., et al. 2006, A\&A, 457, 335

Hekker, S., \& Meléndez, J. 2007, A\&A, 475, 1003

Hinkle, K., Wallace, L., \& Livingston, W. 1995, PASP, 107, 1042

Horner, J., Wittenmyer, R. A., Hinse, T. C., \& Marshall, J. P. 2014, MNRAS, 439, 1176

Huélamo, N., Figueira, P., Bonfils, X., et al. 2008, A\&A, 489, L9

Kaeufl, H.-U., Ballester, P., Biereichel, P., et al. 2004, in Proc. SPIE 5492, eds. A. F. M. Moorwood, \& M. Iye, 1218
Kjeldsen, H., \& Bedding, T. R. 1995, A\&A, 293, 87

Kjeldsen, H., \& Bedding, T. R. 2011, A\&A, 529, A8

Kupka, F., Piskunov, N., Ryabchikova, T. A., Stempels, H. C., \& Weiss, W. W. 1999, A\&AS, 138, 119

Laskar, J., \& Correia, A. C. M. 2009, A\&A, 496, L5

Laughlin, G., Chambers, J., \& Fischer, D. 2002, ApJ, 579, 455

Lee, J. W., Kim, S.-L., Kim, C.-H., et al. 2009, AJ, 137, 3181

Lee, M. H. 2004, ApJ, 611, 517

Lee, M. H., \& Peale, S. J. 2002, ApJ, 567, 596

Lee, M. H., \& Peale, S. J. 2003, ApJ, 592, 1201

Lovis, C., Ségransan, D., Mayor, M., et al. 2011, A\&A, 528, A112

Luck, R. E., \& Challener, S. L. 1995, AJ, 110, 2968

Marcy, G. W., \& Butler, R. P. 1992, PASP, 104, 270

Marcy, G. W., Butler, R. P., Fischer, D., et al. 2001, ApJ, 556, 296

Marois, C., Zuckerman, B., Konopacky, Q. M., Macintosh, B., \& Barman, T. 2010, Nature, 468, 1080

Mayor, M., \& Queloz, D. 1995, Nature, 378, 355

Meschiari, S., Wolf, A. S., Rivera, E., et al. 2009, PASP, 121, 1016

Mitchell, D. S., Reffert, S., Trifonov, T., Quirrenbach, A., \& Fischer, D. A. 2013, A\&A, 555, A87

Niedzielski, A., Konacki, M., Wolszczan, A., et al. 2007, ApJ, 669, 1354

Niedzielski, A., Goździewski, K., Wolszczan, A., et al. 2009a, ApJ, 693, 276

Niedzielski, A., Nowak, G., Adamów, M., \& Wolszczan, A. 2009b, ApJ, 707, 768

Percy, J. R., Wilson, J. B., \& Henry, G. W. 2001, PASP, 113, 983

Press, W. H., Teukolsky, S. A., Vetterling, W. T., \& Flannery, B. P. 1992, Numerical Recipes in FORTRAN, The art of scientific computing, 2nd edn (Cambridge University Press)

Prugniel, P., Vauglin, I., \& Koleva, M. 2011, A\&A, 531, A165

Quirrenbach, A., Reffert, S., \& Bergmann, C. 2011, in AIP Conf. Proc., 1331, eds. S. Schuh, H. Drechsel, \& U. Heber, 102

Reffert, S., \& Quirrenbach, A. 2011, A\&A, 527, A140

Reffert, S., Christoph Bergmann, C., Quirrenbach, A., et al. 2014, A\&A, submitted

Rivera, E. J., Laughlin, G., Butler, R. P., et al. 2010, ApJ, 719, 890

Rothman, L. S., Rinsland, C. P., Goldman, A., et al. 1998, J. Quant. Spectr. Rad. Transf., 60, 665

Sato, B., Ando, H., Kambe, E., et al. 2003, ApJ, 597, L157

Sato, B., Omiya, M., Harakawa, H., et al. 2012, PASJ, 64, 135

Seifahrt, A., \& Käufl, H. U. 2008, A\&A, 491, 929

Tan, X., Payne, M. J., Lee, M. H., et al. 2013, ApJ, 777, 101

Tuomi, M. 2012, A\&A, 543, A52

Valenti, J. A., Butler, R. P., \& Marcy, G. W. 1995, PASP, 107, 966

van Leeuwen, F. 2007, A\&A, 474, 653

Wolszczan, A., \& Frail, D. A. 1992, Nature, 355, 145

Zechmeister, M., Reffert, S., Hatzes, A. P., Endl, M., \& Quirrenbach, A. 2008, A\&A, 491, 531 